\title{
Morphologie und Anatomie des Enteropneusten Saccoglossus pygmaeus Hinr. \& Jac.
}

\author{
Von LUDOLF JACOBI.
}

(Ans der Biologischen Anstalt auf Helgoland.)

Mit 28 Abbildungen im Text.

Inhalt.

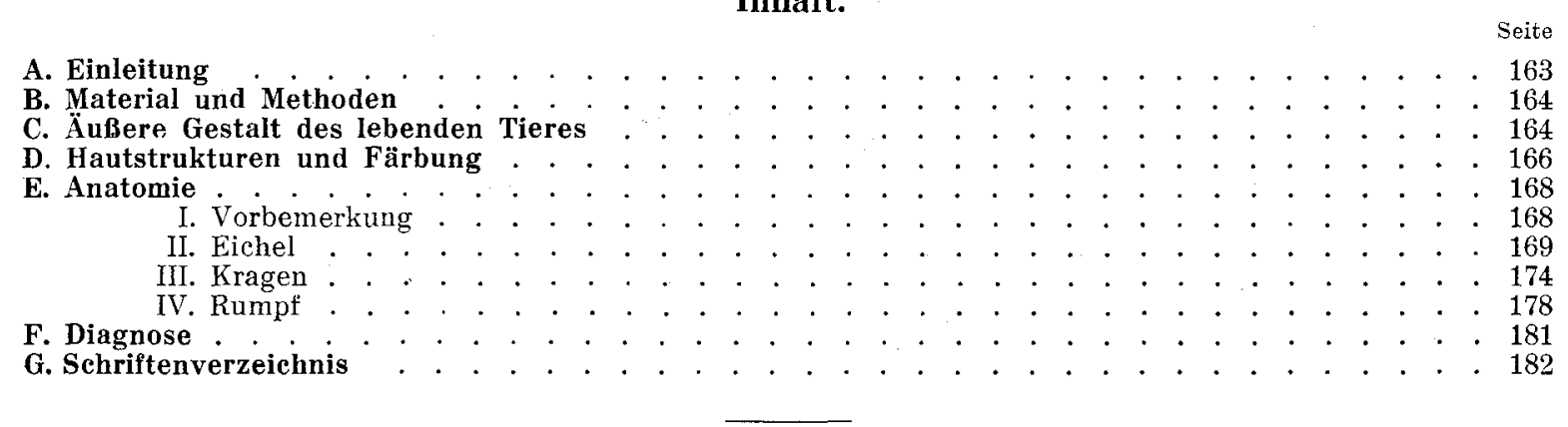

A. Einleitung.

Die Bestimmung eines Enteropneusten bereitet aus folgenden Gründen stets Schwierigkeiten:

1. Die äußere Form und die Farbe der Tiere ist bei verschiedenen Arten wenig verschieden, die Variationsbreite der Individuen einer Art aber ziemlich groß, das einzelne Tier zu erheblichen Formveränderungen befähigt.

2. Die Tiere sind äußerst zerbrechlich. So sind z. B. von manchen Arten die postbranchialen Rumpfregionen unbekannt.

3. Die Fixierung von Enteropneusten ist schwierig (s. Brons S. 391, sowie Abschn. $B$ dieser Arbeit). Fixierte Tiere sind nach ihren äußeren Kennzeichen nicht mehr identifizierbar.

4. Zur Bestimmung fixierter - meist auch lebender - Enteropneusten ist also eine Untersuchung der inneren Organe notwendig, was dadurch erschwert wird, daß die bisher bekannten Arten nicht nach einheitlichen Gesichtspunkten beschrieben sind, und daß vielen Arbeiten eine kurze, trotzdem aber vollständige Diagnose fehlt.

In der vorliegenden Arbeit ist versucht worden, eimmal das lebende Tier so genau wie möglich zu beschreiben, da es bei Saccoglossus pygmaeus möglich ist, nach den äußeren Merkmalen des lebenden Tieres die Art zu bestimmen. Andererseits habe ich mich bemüht, den anatomischen Bau von $S$. pygmaeus so vollständig, kurz und übersichtlich wie möglich darzustellen. Sehr erleichtert wurde mir die Arbeit dadurch, daf Herr Professor Dr. VAN DER FIORST (Johannesburg) so freundlich war, mir ein Manuskript seiner Arbeit: „Beschreibung einer hypothetischen Saccoglossus-Art (Bronn's Klassen und Ordnungen des Tierreichs. Im Druck) zugehen zu lassen. Die Diagnose von Saccoglossus pyg!maeus (Abschnitt $\mathrm{F}$ der vorliegenden Arbeit) habe ich der Beschreibung van DER Horsts 
so weit wie möglich angeglichen. Herrn Professor VAN DFR HORST möchte ich an dieser Stelle für seine Freundlichkeit meinen besten Dank aussprechen.

Der Biologischen Anstalt auf Helgoland, ganz besonders Herrn Professor Dr. HaGMEIER, der mir die Anregung zu dieser Arbeit gab, und der ihre Fertigstellung ermöglichte, möchte ich vielmals danken. Herrn Oberpräparator HinRICHs und Herrn Fischmeister HoLtmann danke ich für die Hilfe bei der Materialbeschaffung.

\section{B. Material und Methoden.}

Saccoglossus pygmaeus bewohnt den Helgoländer Amphioxusgrund (HinRICHS und JacoBI 1938). Am sichersten bekamen wir ihn mit dem Hensenschen Bodeneiernetz. Die Tiere sind äußerst empfindlich, ganz unverletzte Tiere bekamen wir so nicht. Da die Tiere sehr regenerationsfähig sind, hielt ich sie längere Zeit lebend, und zwar in Röhrengläsern, die einige cm hoch mit Amphioxussand gefüllt waren und in durchströmten Aquarien standen, Dabei muß man darauf achten, daß das Wasser nicht zu warm wird, ferner darauf daß sich in den Gläsern nicht zu viel Detritus ansammelt. Treten im Sand schwarze Stellen auf, so kommen die Tiere vermutlich aus $0_{2}$-Mangel heraus und gehen ein. Sie können dann auch an der Glaswand ihres Gefäßes hochkriechen. Es besteht also die Gefahr, daß die Tiere aus ihren Gläsern entweichen. Fühlen sie sich aber wohl, so kommen sie nicht aus dem Sande heraus und regenerieren fehlende Teile sehr schnell. Genauere Daten über die Regeneration fehlen mir noch.

Als Fixierungsflüssigkeiten dienten Bouin, Zenker, Helly und Carnoy. Irgendwelche nachteiligen Wirkungen der Sublimatgemische habe ich nicht wahrnehmen können. Einige unangenehme Folgen hatte Carnoysche Flüssigkeit: Die Eichelhöhle war bei beiden Tieren, die ich mit Carnoy fixierte, fast völlig verschwunden; die Eichelorgane, um die normalerweise ein beträchtlicher Hohlraum ist (Abb. 8-10), berührten direkt das die Eichelhöhle von der Längsmuskulatur trennende Bindegewebe. Der Eicheldarm zeigte bei beiden Tieren einen abnorm gewundenen Verlauf, was wohl mit der Schrumpfung der Eichelhöhle zusammenhängt.

Wurden die Tiere ohne Narkose fixiert, so kontrahierten sie sich, auch wenn man die Fixierungsflüssigkeit heiß anwandte, stark. Am geringsten war noch die Verkürzung der Eichel, die $1 / 3$ bis höchstens $1_{2}$ ihrer ursprïnglichen Länge verlor. Ihre Dicke nahm dafür etwas zu. Der Eichelstiel verschwand völlig in der Vorderseite des Kragens. Letzterer wurde nach einfacher Fixierung etwa auf die Hälfte verkürzt gegenüber dem des lebenden Tieres. Mindestens ebenso stark, vermutlich jedoch noch stärker als der Kragen rerkürzte sich der Rumpf, was jedoch, da sich das Tier dabei sehr stark aufknäuelte, nicht mefbar war.

Die Form des Tieres blieb besser erhalten, wenn es nach vorsichtiger Narkose fixiert wurde. Als Narkotika dienten $70^{\circ}$ iger Alkohol, mit Chloroform gesättigtes Seewasser oder $0,5^{\circ} 0_{0}$ ige Lösung von Äthylurethan in Seewasser. Die Narkotisierungsflïssigkeit wurde zu den in einer Petrischale mit reinem Seewasser befindlichen Tieren nach and nach zugetropft. Reagierte das Tier auf starke taktische Reize der Eichelspitze nicht mehr, wurde es fixiert. Wurde nun die Fixierungsflüssigkeit kalt angewandt, so kontrahierten sich die Tiere, vor allen Dingen Kragen und Rumpf, immer noch sehr stark. Anders, wenn man die Fixierungsflüssigkeit sehr heiß (fast kochend) anwandte. Letzteres hatte freilich wieder mehrere Nachfeile: So war bei sümtlichen mit heißer Lösung fixierten Tieren im Kragen - bei den nreisten auch im Rumpf - die Epidermis von ihrer Grenzmembran abgerissen. Die inneren Organe der Tiere waren kontrahiert wie nach einfacher Fixierung. Die Eichelorgane waren meist von dem basalen Epithel losgerissen und nach vorn verschoben. Dieses Fixierungsergebnis kam dadurch zustande, daß die Tiere bei der Berührung mit der heißen Lösung sich ruckartig kontrahierten, so daß die Epidermis nicht folgen konnte. Ferner stülpten die Tiere bei Fixierung mit heißer Lösung vielfach Teile der Kiemenzungen (Abb. 3 KZ) aus den Poren heraus, was die anatomische Untersuchung erschwerte.

Die Eichel bleibt nach Alkoholnarkose, auch wenn die Fixierungsflüssigkeit kalt angewandt wird, mindestens so lang wie beim lebenden Tier. Ihr Volumen wird durch den Alkohol - z. T. wohl dadurch, daß die Epidermisschleimzellen quellen (Abb. 8-10) - deutlich vergrößert (vgl. Abb. 2 mit 2). Kragen und Rumpf verkürzen sich dagegen nach Alkoholnarkose und darauf folgender Fixierung mit kalter Lösung sehr stark; die Rumpfepidermis wird durch lange Alkoholeinwirkung zerstört.

Die besten Ergebnisse erzielte ich, wenn ich die Tiere kurze Zeit ( $1 / 2$ Stunde) mit Chloroformseewasser narkotisierte. Dabei muß man freilich das Tier stets beobachten, da bei einem gewissen Sättigungsgrad des Seewassers mit Chloroform die Rumpfepidermiszellen zerfallen, ein Vorgang, der sich deutlich verfolgen läßt. Auch wenn sich die Tiere sehr lange in stark verdünnter Chloroformseewasserlösung befinden, verändert sich ihre Epidermis. Da letzteres auch bei Tieren eintrat, die ich einige Stunden bei Zimmertemperatur beobachtete - die Untersuchungen habe ich fast alle im Winter gemacht -, bin ich nicht ganz sicher, ob das Chloroform in verdünnter Lösung wirklich schädigend wirkt.

Die fixierten Tiere wurden über Alkohol - Methylbenzoat-Zelloidin - Benzol (Romeis 1928), (bisweilen auch Alkohol - Xylol) in Paraffin eingebettet, die Schnittdicke betrug 5-7 $\mu$. Günstig ist, daß $S$. pygmaeus, obwohl er ziemlich reinen Sand bewohnt, kein ausgesprochener Sandfresser ist. Nur vereinzelt finden sich im Darm Sandkörner, können hier freilich dadurch, daß das Tier so klein und stets aufgeknäuelt ist, sehr stören.

Als Färbemittel wurden verwandt: Toluidin - Erythrosin - Orange GG nach DomINIcI und TischutKIN (ROMEIS 1932), (TE0), Eisenhämatoxylin nach HEIDENHAIN (HEH) oder WEIGERT, DELAFIELDSches Hämatoxylin, Azanfärbung nach HEIDENHAIN.

\section{C. Äußere Gestalt des lebenden Tieres.}

S. pygmaeus wird kaum über $30 \mathrm{~mm}$, seine Eichel bis $7 \mathrm{~mm}$ lang. Bei ausgewaehsenen, geschlechtsreifen Tieren ist letztere meist relativ (oft auch absolut) kürzer als 
bei jungen. Nahe ihrer Basis ist die Eichel am dicksten (gut $1 \mathrm{~mm}$ ). Der Eichelquerschnitt ist bei dem fixierten Tier rund (s. Abb. 8-10), beim lebenden, in einer Glasschale kriechenden, beobachtete ich bisweilen eine schwache, dorsoventrale Abplațtung. Auf ihrer dorsalen Seite zeigt die Eichel eine feine Längslinie (Abb. 4 W. D.), die durch die Hautstrukturen zustande kommt, und die nach Fixierung vielfach als Furche erscheint (Abb. 8-10 W. D.).

Der Eichelstiel ist an seiner dünnsten Stelle $0,15-0,24 \mathrm{~mm}$ dick. Etwas vor dieser Stelle ist auf der linken Seite der Eichelporus (Abb. 4 E. P.) sichtbar, um den herum vielfach eine besonders intensive Flimmerbewegung erkennbar ist.

Der Kragen ist $0,45-1,25 \mathrm{~mm}$ lang, gestreckt bis doppelt so lang wie kontrahiert. Er wird bis $1,3 \mathrm{~mm}$ breit. Auch gestreckt ist er fast stets breiter als lang. Der Querschnitt des Kragens ist annähernd rund, jedoch zu mannigfachen Formänderungen befähigt. In seinem vorderen Teil ist der Kragen ringförmig eingedellt; hinter dieser Delle erreicht er seinen größten Umfang, verjüngt sich caudalwärts wieder, so in den Kiementeil ohne Andeutung eines Operculums übergehend. Kontrahiert können jedoch die hinteren Teile des Kragens die vorderen der Kiemenregion überdecken. Im hinteren Teil des Kragens, meist an seiner dicksten Stelle, befindet sich die „typische Ringfurche" (Abb. 4 K. F.), die stets erkennbar ist, wenn sie auch meist beim gestreckten Kragen nur sehr schwach ausgebildet ist. Sie

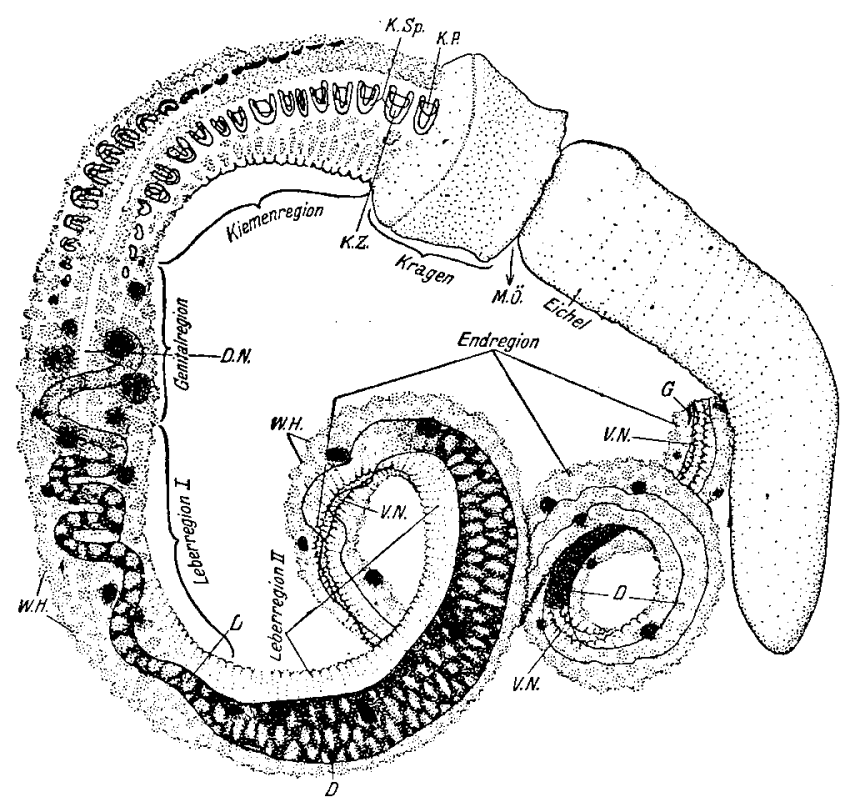

Abb. 1. S. pygmaeus, reifes $0^{*}$, nach dem Leben gezeichnet Vergr. 20 fach.

$\mathrm{D}=$ Darm, $\mathrm{DN}=$ dorsaler Nervenstamm, $\mathrm{KP}=$ Kiemenporus, $\mathrm{KSp}=$ Kiemenspalte, $\mathrm{KZ}=$ Kiemenzunge, $\mathrm{MO}=$ Mundöffnung, VN = ventraler Nervenstamm, $\mathrm{WH}=$ Warzenhaufen.

Die roten Punkte $(G)$ kennzeichnen das Tier als $\sigma^{r}$. Nach Hinrichs und Jacobi 1938. wird bisweilen dadurch, daß in ihr die Pigmentkörnchen (s. Kap. D) fehlen, oder dadurch, daß die Pigmentkörnchen etwas vor ihr besonders dicht liegen, verdeutlicht. Die Mundöffnung ist wie bei allen Enteropneusten sehr groß, durch Einfaltung der sie umgebenden Kragenpartieen kann sie weitgehend geschlossen werden.

Der Rumpf des geschlechtsreifen Tieres wird 10-20 mm lang, davon entfallen auf die Kiemenregion 1,5-3, auf die Leberregion 3,5-7,5 und auf die Endregion 2,5-9 mm. Die Genitalregion - d. h. die Region, in der der Darm als Ösophagus den Pharynx mit dem Leberdarm verbindet - ist sehr kurz (Abb. 1), äußerlich meist nicht erkennbar, aber (vergl. Abschn. E) vorhanden. Die Länge der einzelnen Regionen ist sowohl absolut als auch aufeinander bezogen großen Schwankungen unterworfen. Der Rumpf ist meist, nach Fixierung stets, ventralwärts eingerollt, wodurch die Ventralseite geringelt erscheint (Abb. 1 u. 2). Die ventrale Körpermuskulatur bildet kein erhabenes Muskelband.

Der dorsale Nervenstamm ist in der Kiemen-, Genital- und vorderen Leberregion sehr deutlich (Abb. 1 und 4 D. N.), weiter hinten verschwindet er. Dafür wird der ventrale Nervenstamm (V. N.), der in der Kiemenregion kaum sichtbar ist, caudalwärts deutlicher. Besonders charakteristisch für $S$. pygmaeus ist die Lage seiner Gonaden (Abb. 1, 2 und 28). Die Kiemenregion ist fast völlig frei von Gonaden, während alle übrigen Rumpfregionen - auch die der kleinen Tiere - Gonaden besitzen. Ventral der letzten, nicht mehr typischen Kiemenspalten finden sich die ersten Gonaden, nur bei einem von 24 Tieren reichten sie weiter nach vorn, bis etwa in die Mitte der Kiemenregion. Direkt hinter der Kiemenregion sind die Gonaden meist am zahlreichsten und größten. Bei den $0^{x} \sigma^{x}$ erscheinen sie als leuchtend rote, sehr verschieden geformte Flecke, oft auch als große Haufen roter Pünktchen (Abb. 1). Bei den als große, zur Fortpflanzungszeit (November bis Dezember) bis $0,3 \mathrm{~mm}$ messende, im auffallenden Licht weiß erscheinende Eier erkennbar (Abb. 2). Auch außerhalb der 


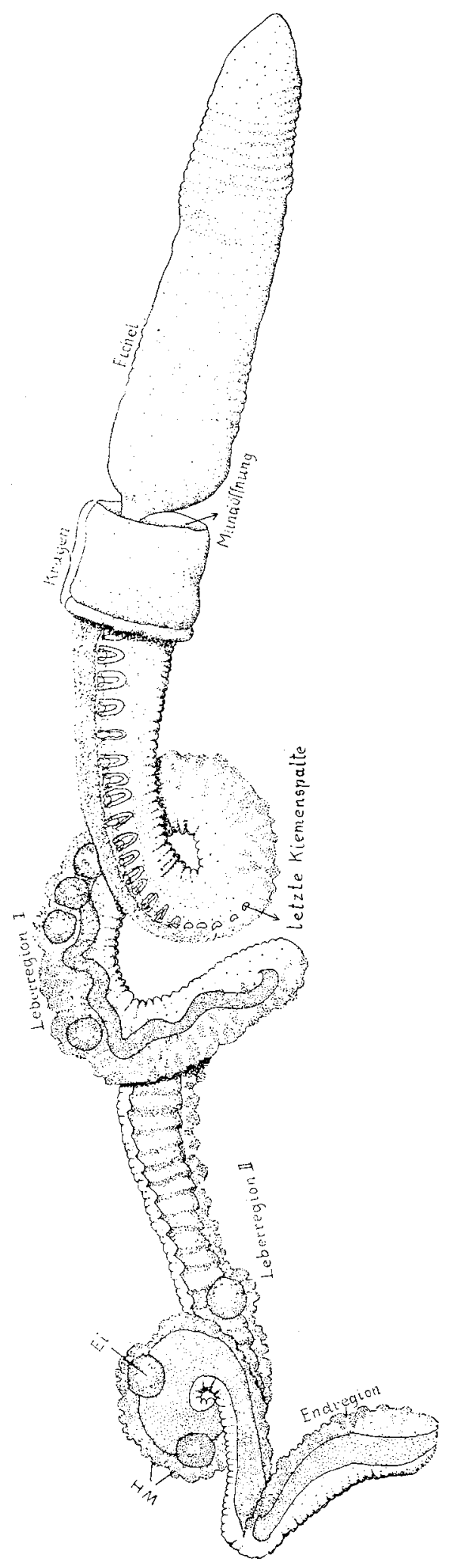

Abb. 2. S. plygmaeus, reifes o zur Fortpflanzungszeit. Vergr. 20 fach. $\mathrm{WH}=$ Warzenhaufen.
Fortpflanzungszeit sind $\sigma^{\pi} \sigma^{x}$ und $\varnothing \propto$ an ihren Gonaden leicht voneinander unterscheidbar, nur ganz kleine Tiere lassen nichts von Gonaden erkennen.

Die Kiemenregion ist vorn 0,5 bis fast 1 , hinten 0,45 bis $0,7 \mathrm{~mm}$ dick. Die Kiemenspalten sind gut sichtbar, es sind 9-22 Paar. Ihre Form und GröBe ist auf Abb. 4 erkennbar. Die Poren (KP) sind sehr verschieden geformt; ihre Dorsalkanten sind oft schwer erkennbar, sodaß das sie umgebende Epithel direkt in das Zungenepithel überzugehen scheint. An den die Spalten begrenzenden Flächen der Septen und Zungen ist stets ein reger Wimperschlag in craniocaudaler oder umgekehrter Richtung erkennbar, der auf den beiden Organen stets in einander entgegengesetzter Richtung verläuft.

Gegen die Genitalregion, in der noch die letzten - nicht mehr typischen - Kiemenspalten liegen, ist die Kiemenregion häufig durch eine die Dorsalseite des Tieres quer durchziehende Furche getrennt. Solche Furchen kommen vielfach auch weiter hinten - bis in die vordere Leberregion hinein - vor. Sie teilen dann die "Genitalhöcker" roneinander ab. Jedenfalls ist es wahrscheinlich, daß sie durch die Auftreibung des Körpers durch die Gonaden bedingt sind. Sind in der Genitalregion keine Gonaden vorhanden, dann sind auch die Höcker nicht ausgebildet (Abb. 2). Bei kleinen reifen Tieren dagegen, die in der Genitalregion die weitaus meisten Gonaden besitzen, erscheint der Rumpf jederseits des dorsalen Nervenstammes sehr stark aufgetrieben, im Querschnitt fast von der Form eines gleichseitigen Dreiecks, dessen Spitze (Ventralseite des Tieres) freilich tief abgeschnitten und dessen Ecken gerundet sind.

Direkt hinter der Kiemenregion wird der Rumpf reifer Exemplare von $S$. pygmaeus etwas dicker $(0,6-0,7 \mathrm{~mm})$, dann - bereits im ersten Teil der Leberregion - kontinuierlich dünner (bis etwa $0,5 \mathrm{~mm})$. Im zweiten Teil der Leberregion wird er meist wieder etwas dicker $(0,6 \mathrm{~mm})$. In der Endregion ist der Tierkörper normalerweise am dünnsten $(0,3-0,4 \mathrm{~mm})$. Der Darm ist in der Genitalregion makroskopisch schwer erkennbar. In beiden Leberregionen ist er intensiv grün bis bräunlichgrün gefärbt. Im ersten Teil ist er dünn und stets intensiv geschlängelt, im zweiten dick und gerade oder geschlängelt. In der Endregion ist der Darm schwach grün, fast farblos, und wenig gewunden. Sämtliche Schlängelungen des Darms liegen horizontal, sodaß der Darm bei Betrachtung des Tieres von der Seite gerade erscheint.

\section{Hautstrukturen und Färbung.}

Eichel, Eichelstiel und Kragen von $S$. pygmaeus sind rundherum gleichmäßig bewimpert. Ventralseits ist auch der ganze Rumpf bewimpert. Die dorsalen Teile der Kiemenregion sind - abgesehen natürlich von dem dorsalen Nervenstamm wahrscheinlich wimperfrei, zum mindesten wie die 


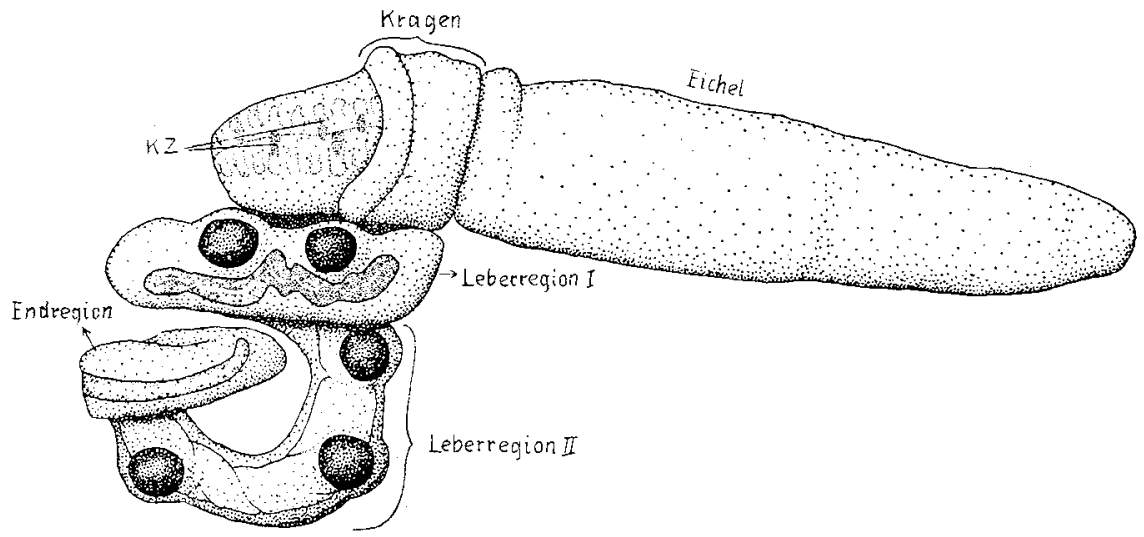

Abb. 3. Dasselbe Tier wie in Abb. 2. 1\%: Std. nark. Alkohol, fix. mit fast kochendem Bouin, aufgehellt mit Xylol. Vergr. 20 fach. $\mathrm{KZ}=$ infolge der heißen Fixierung ausgestülpte Teile der Kiemenzungen.

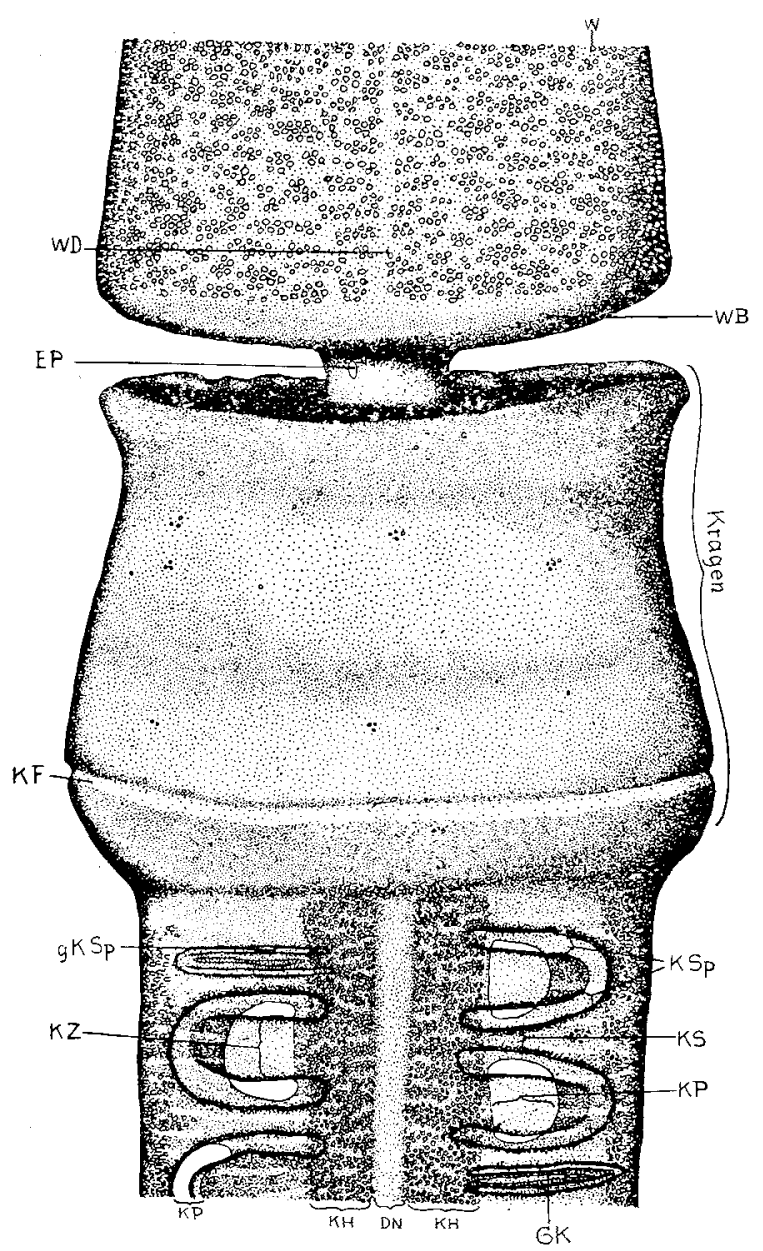

Abb. 4. S. pygmaeus. Basaler Teil der Eichel, Kragen und Beginn der Kiemenregion. Vergr. 67 fach. $\mathrm{DN}=$ dorsaler Nervenstamm, $\mathrm{EP}=$ Eichelporus, GK und gKSp = geschlossene Kiemenspalte, $\mathrm{KF}=$ "typische Ringfurche“, $\mathrm{KH}$ $=$ Kiemenhöcker, $\mathrm{KP}=$ Kiemenporus, $\mathrm{KS}$ $=$ Kiemenseptum, KSp $=$ Kiemenspalte, $\mathrm{KZ}$ $=$ Kiemenzunge, $\mathrm{W}=$ Warzen, $\mathrm{WB}=$ warzenfreier Basalteil der Eichel, WD = warzenfreie Dorsallinie.

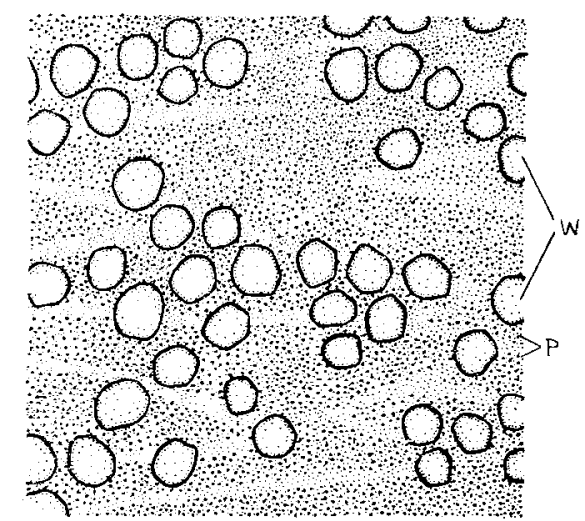

Abb. 5. Eicheloberfläche des lebenden Tieres. Vergr. 400 fach. $W=$ Warzen, $\mathrm{P}=$ Pigmentkörnchen.

der hinteren Regionen wimperarm. Nur ganz selten konnte ich auf den dorsalen Teilen der hinteren Rumpfregionen mit Sicherheit Wimperbewegung feststellen.

Eins der auffälligsten Merkmale von S. pygmaeus, das auch kleine Bruchstücke (z. B. Teile der Eichel) als unserem Tier gehörend erkennen läßt, ist die $\mathrm{Be}-$ warzung seiner Körperhaut (Abb. 4 und 5 W). Die Warzen erscheinen von oben gesehen rund, im optischen Schnitt birnförmig. Sie färben sich mit Neutralrot sehr schnell leuchtend rot, während die sie umgebenden Partien farblos bleiben. In der Eichelepidermis fehlen die Warzen niemals, meist sind sie hier sehr zahlreich und regellos verteilt. Nur ganz an der Basis, sowie auf der bereits beschriebenen (Seite 165) feinen Linie längs der Dorsalseite der Eichel (Abb. 4 WD) sind sie nicht vorhanden. Dem Kragen fehlen sie fast völlig; die wenigen, die hier $\mathrm{zu}$ sehen sind, sind erheblich kleiner als die der Eichel. 
In der Kiemenregion ist die Anordnung der Wärzchen besonders typisch (Abb. 1, 2 und 4): Die Partieen um die Kiemenporen, der dorsale Nervenstamm (DN) und die ventrale Epidermis sind unbewarzt. Jederseits des Dorsalnervenstammes ist die Körperhaut außerordentlich dick und fast stets sehr stark bewarzt, so über den Kiemenspalten zWei Längswülste $(\mathrm{KH})$ bildend. Letztere werden cranialwärts immer dicker und undurchsichtiger; am Beginn des Kragens erscheinen sie plötzlich abgeschnitten. Auch ventral der Kiemenporen ist die Epidermis ziemlich stark bewarzt. Die Wärzchen liegen hier entweder in Haufen zusammen (wie auf den hinteren Rumpfregionen), oder sie sind regellos zerstreut. Ventralwärts werden sie spärlicher, auf der Bauchseite fehlen sie.

In den hinteren Rumpfregionen liegen die Warzen stets in Haufen zusammen (Abb. 1 und $2 \mathrm{WH}$ ). Letztere ragen etwas über das umgebende Epithel hinaus. Die Ventralseite des Rumpfes ist stets unbewarzt; in der Leberregion sind auch große Teile der Lateralseiten warzenfrei, während in der Genital- und Endregion die Warzenhaufen weit ventralwärts reichen.

Durch die Fixierung quellen die bewarzten Partien meist ganz außerordentlich: So erscheint die Eicheloberfläche beim fixierten Tier genetzt, die Netzmaschen besitzen einen größeren Durchmesser $(10-25, \imath)$ als die Wärzchen $(7-14, \imath)$. Die im optischen Schnitt durchschimmernde Eichelepidermis ist beim fixierten Tier dicker als beim lebenden. Dieses, sowie die Ergebnisse der histologischen Untersuchung der Eichelepidermis machen es wahrscheinlich, daß die Warzen Schleimvakuolen sind, die durch das Fixierungsmittel zur Quellung gebracht werden. In der Kiemenregion sind die Verhältnisse weniger klar: Nach Fixierung ist hier die Epidermis dorsal der Kiemenspalten sehr stark verquollen. Der Dorsalnervenstamm erscheint dadurch als tiefe Rinne (Abb. 23). Auch die die Kiemenporen umgebenden Epidermisteile bestehen aus verquollenen Schleimzellen. Es müssen hier also Schleimvakuolen vorhanden sein, die beim lebenden Tier nicht als Warzen erscheinen, sich auch nicht mit Neutralrot färben lassen, die sich histologisch aber nicht ohne weiteres von den Warzen unterscheiden lassen. Die starke Kontraktion der Kiemenregion bei der Fixierung kompliziert - zumal, wenn. gleichzeitig die Schleimvakuolen sehr stark quellen - das ganze natürlich sehr.

Die Färbung der Eichel von S. pygmaeus ist milchig-hellgelb, basal, wo die Warzen fehlen, dunkler. Die Eichel ist der dichteste und undurchsichtigste Körperteil. Der Kragen ist stets dunkler als die Eichel, vielfach ist er hellbraun, bei kleineren Tieren dunkelgelb. Verschiedene Kragenzonen sind nicht vorhanden, bei manchen Tieren ist aber der hintere Abschnitt des Kragens dunkler als der vordere. Die Kiemenregion ist wieder etwas heller als der Kragen, nach hinten wird sie kontinuierlich farbloser. Die Epidermis der postbranchialen Rumpfregionen ist fast farblos, der Darm dadurch dort sehr gut erkennbar. Die Färbung wird durch kleine braune Pigmentkörnchen verursacht, die beim lebenden Tier sehr deutlich sichtbar sind (Abb. 5 P).

\section{E. Anatomie.}

\section{Vorbemerkung.}

Da die Kenntnis der Lage der Eichel- und Kragenorgane der Enteropneusten für das Verstïndnis des Folgenden notwendig ist, möchte ich eine kurze Beschreibung der bei Enteropneusten herrschenden Verhältnisse geben, genameres ist bei SPENGEL (1893) und VAN DER HORST (Bronn, 1927-1934) zu finden. Die Eichel besteht - das ganz besonders bei Saccoglossus-Arten - zum weitaus größten Teil aus M uskulatur und Eichelhöhle, die Eichelorgane finden sich nur ganz an der Basis, reichen nur so weit nach vorn, wie dies auf $\mathrm{Abb}$. $6 \mathrm{zu}$ sehen ist. Der Epidermis in nen anliegend findet sich eine dünne. Ringmuskelschicht, von dort nach innen zu ein mächtiger Längsmuskel, der als die sehr dicke Wand eines Hohlzylinders die Eicheihöhle umgibt.

In den basalen Teil der Eichelhöhle ragen die aus dem Kragen kommenden Eichelorgane hinein. In ihrem Bereich wird die Eichelhöhle durch Septen in zwei laterale Teile geteilt, diese werden ihrerseits wieder durch die caudalwärts sich verbreiternden Eichelorgane in je einen dorsalen und ventralen Teil geschieden. Die dorsalen Teile, oder - z. B. bei S. pygmaeus - einer derselben, münden mittels eines Porus (Abb. 4 und 15 EP) nach außen.

Die Eichelorgane (vgl. dazu Abb. 6) sitzen dem Ei cheldarm auf, der als Blindsack der Mundhöhle in die Eichelhöhle hineinragt. Im Eichel hals verläuft der Eicheldarm dorsal des E i chelskeletts. Über das Vorderende des Skeletts, die sog. Endplatte, greift der Ficheldarm mittels einer bei S. pygmaeus sehr gering entwickelten ventralen „Bli i d t a s ch e“. Diese Blindtasche liegt der Endplatte flächenhaft auf. Weiter cranialwärts verjüngt sich der Eicheldarm nach und nach, und zwar vor allem ventral, sodaß die Blindtasche verschwindet. Der im Eichelhals verlaufende Teil des Eicheldarms wird als Eicheldarmhals, die Bliadtasche und die cranial dieser Tasche gelegenen Teile des Eicheldarms als Eicheldarmkörper bezeichnet. 
Dem Eicheldarmkörper liegt dorsal die Herzblase auf, zwischen Eicheldarmkörper und Herzblase befindet sich der zentrale Blutraum. Eicheldarmkörper und Herzblase werden teilweise vom Glomerulus überkleidet.

Das Eichelskelett verläuft als unpaarer Skeletteil im Eichelhals, sein Vorderende bildet die bereits beschriebene Endplatte. Er ist ferner auf eine kurze Strecke in einen dorsalen Skelettk örper und einen ventralen $\mathrm{Kiel}$ geteilt. Lateral des unpaaren Skeletteils finden sich die abführenden Eichelgefäße, sowie die bis fast zur Skelettendplatte in den Eichelhals reichenden Kragencölome. Im Kragen, cranial der Einmündungsstelle des Eicheldarms in die Mundhöhle, teilt sich das Eichelskelett in die Schenkel, die die Mundhöhle ventralwärts teilweise umgreifen.

Die in der Epidermis der Eichelbasis sehr-mächtige Nervenschicht (Abb. 6) ist im Eichelstiel nur dorsalseits zu finden, die Epidermis erinnert dort auf Querschnitten (Abb. 15) schon sehr an das Kragenmark, in das sie übergeht.

Ventral des Kragenmarks verläuft das D orsalgefä $\beta$, das das Blut aus Kragen und Rumpf in den Sinus renosus führt, von wo es in den zentralen Blutraum gelangt. Aus dem zentralen Blutraum strömt das Blut in die Glomerulusgefäße, von dort z. T. durch die abführenden Eichelgefäße in den Kragen und den Rumpf zurück z. T. cranialwärts in die Eichel. Dem Dorsalgefäß des Kragens anliegend finden sich die bzw. der Perihämalraum, der eine Ausstülpung des Rumpfeöloms ist.

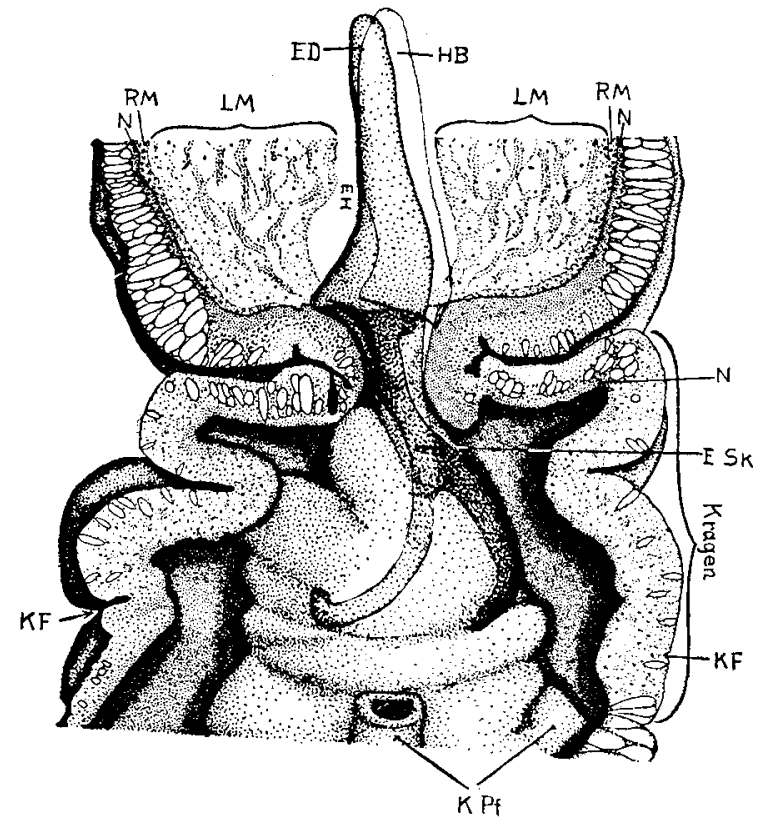

Abb. 6. Rekonstruktion des Darmabschnitts in Eichel und Kragen, von dorsolateral gesehen. Umrisse der Herzblase (HB) und des Eichelskeletts (ESk) rot, Eichelmuskulatur (LM und RM), Eichel- und Kragenepidermis im Längsschnitt.

Fix. Bouin, Fbg. TEO, Vergr. 67 fach.

$\mathrm{ED}=$ Eicheldarm, $\mathrm{EH}=$ Eichelhöhle, KF $=$ „typische Ringfurche“", KPf $=$ Kragenpforten,

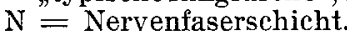

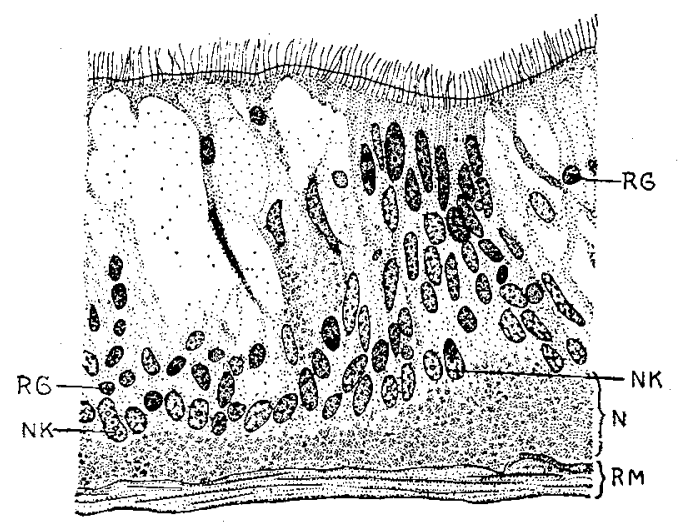

Abb. 7. Querschnitt durch die warzenfreie Dorsallinie der Eichel. Nark. Chloroform, fix. Zenker, Fbg. TEO, Vergr. 603 fach. $\mathrm{N}=$ Nervenfaserschicht, $\mathrm{NK}==$ Kerne des Nervensystems (?), $R G=$ Granula (s. Text), RM = Ringmuskelschicht.

\section{Eichel.}

Epidermis: Die Eichelepidermis ist normalerweise rundherum - beim lebenden Tier auch wohl in der warzenfreien Dorsallinie - annähernd gleich dick $(60-70 \mu)$. Sind die Schleimvakuolen extrem gefüllt und gequollen (Abb. 8-10), so ist sie natürlich noch höher, indeß die Dorsallinie als Kerbe erscheint. Die Nervenfaserschicht ist durchschnittlich $2-3 \mu$ dick, ihre Dicke variiert aber; so wird sie z. B., wenn die Schleimvakuolen extrem gefüllt sind und bei der Fixierung noch quellen, zusammengedrückt. Längs der warzenfreien Dorsallinie (Abb. 7) ist die Nervenfaserschicht - vor allem in den hinteren Teilen der Eichel - ein wenig verdickt. Unter dem distalen Grenzsaum ist ein feiner Cytoplasmasaum sichtbar, in dem die Basalkörner der sehr zarten ca. $4 \mu$ hohen Wimpern liegen. Letztere erscheinen auf der Dorsallinie besonders deutlich. 
Charakteristisch für die Eichelepidermis von $S$. pygmaeus ist, daß sie nur eine Art von Drüsen besitzt, nämlich die sich mit Toluidin meist intensiv färbenden Schleimdrüsen (Abb. 8-10). Letztere fehlen nur auf der warzenfreien Dorsallinie (Abb. 7). Vereinzelt fand ich noch relativ große (kleiner als Zellkerne), runde, sich mit Eosin oder Orange-Erythrosin homogen rot färbende Körper (Abb. $7 \mathrm{RG}$ ), die vielleicht Reste reduzierter Drüsen sind.

Die Zellkerne liegen in der Eichelepidermis größtenteils basal, zwischen der Nervenschicht und den Schleimvakuolen eine mehr oder weniger dicke und dichte, des öfteren unterbrochene Lage bildend. Distal sind zwischen den Schleimvakuolen auch stets einige Kerne zu finden. In der Dorsallinie (Abb. 7) ist die Epidermis besonders kernreich, die Kerne dringen hier sehr weit distalwärts vor.

Zellgrenzen konnte ich mit meinen Methoden nirgendwo in der Epidermis von S. pygmaeus sichtbar machen, ich kann also hinsichtlich der Zugehörigkeit bestimmter Kerne zu bestimmten Zelien (z. B. Nerven-, Wimper-, Drüsen-, Sinneszellen, „Membrana reticulata" u. a.) nichts sicheres sagen. Daß die in den Abb. 7, 16 und 18 mit NK bezeichneten Kerne (vgl. Kragenmark) dem Nervensystem angehören ist freilich wahrscheinlich.

Ganz an der Basis der Eichel wird die Nervenfaserschicht sehr dick (Abb. 6). Die Epidermis ist hier sehr kernreich, während Schleimvakuolen selten sind (beim lebenden Tier sind ja auch keine Warzen vorhanden). Im Eichelstiel verschwinden ventral die großen Nervenmassen vollkommen, während die dorsale Epidermis hier fast in jeder Hinsicht an das Kragenmark, in das sie größtenteils übergeht, erinnert (Abb. 15). Die ventrale, skelettbildende Eichelstielepidermis geht in das entsprechende Epithel der Mundhöhle über, dort die Wimpern verlierend.

Muskulatur: Die Ringmuskelschicht der Eichel ist $3-9 \mu$ dick, meist etwas dicker als die Nervenfaserschicht. Sie besteht aus mehreren sehr dünnen, parallel verlaufenden Fasern, zwischen denen sehr wenig Kerne - oft auf einem ganzen Querschnitt kein einziger - liegen (Abb. 7). Einen Sphinkter an der Eichelbasis bildet sie nicht.

Die Längsmuskulatur der Eichel ist sehr kräftig und läßt der Eichelhöhle nur wenig Platz. Sie zeigt niemals eine konzentrische Schichtung (Abb 8-10 LM). Die einzelnen Längsmuskelfasern sind ziemlich dick, aber auf Längsschnitten nicht allzu weit zu verfolgen. Sie sind vor ihrem Ende stets in viele feine Fasern aufgespalten, sodaß sich ihre

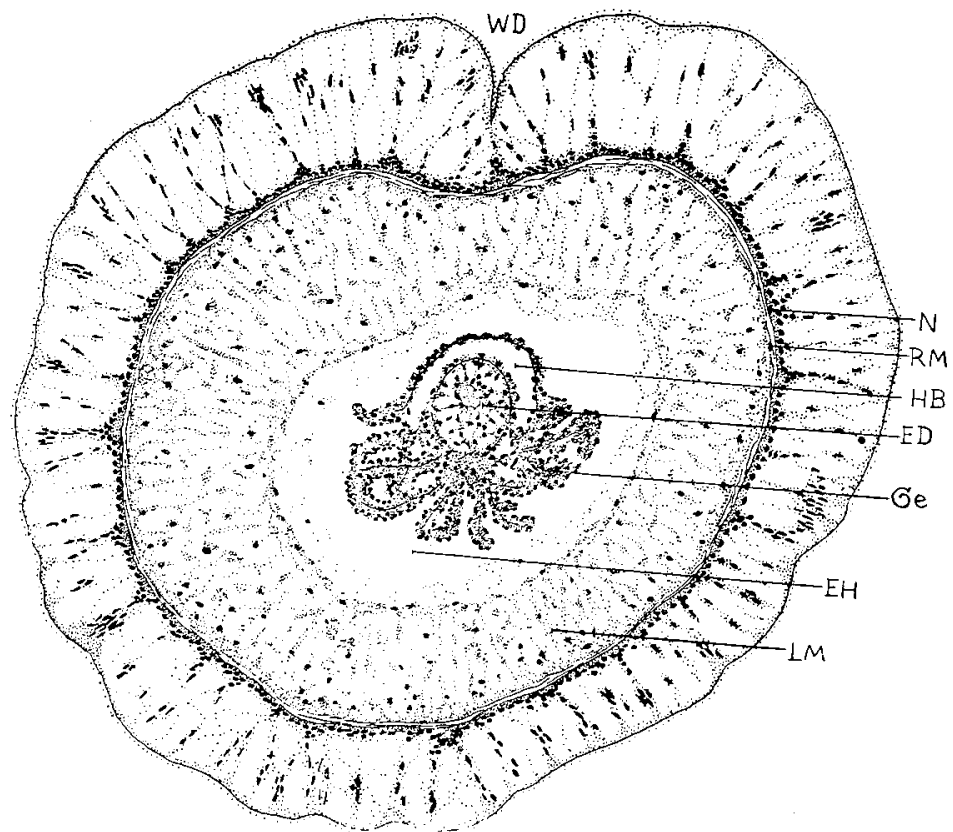

Abb. 8. Querschnitt durch den basalen Teil der Eichel, $200 u$ hinter Beginn der Eichelorgane. Nark. Alkohol, fix. Bouin, Fbg. HEH, Vergr. 105 fach.

$\mathrm{ED}=$ Eicheldarm, $\mathrm{EH}=$ Eichelhöhle, $\mathrm{Ge}=$ Glomerulus, $\mathrm{HB}=$ Herzblase, $\mathrm{L} M=$ Längsmuskulatur, $\mathrm{N}=$ Nervenfaserschicht, RM = Ringmuskelschicht, WD = warzenfreie Dorsal linie.
Ursprungs- und Ansatzstellen schwer feststellen lassen(Abb.6). Mit den die Eichelhöhle umrundenden Fasern, untereinander, mit den äußeren Ringmuskelfasern und mit dem Bindegewebe sind sie durch zahlreiche, radial verlaufende Fasern verbunden. Letztere sind besonders im basalen Teil der Eichel, in dem sich die Längsmuskelfasern dem Centrum der Eichel zuwenden (Abb. 6), deutlich. Auf der äußeren und inneren Grenze der Längsmuskelschicht findet sich ein besonders dichtes, vielfach auch kernreiches, faseriges Gewebe. Spezifische Unterschiede zwischen Muskel- und Bindegewebsfasern konnte ich nicht feststellen.

In den Eichelstiel dringt keinerlei Eichelmuskulatur ein (Abb. 11).

Eichelhöhle: Die Eichelhöhle erstreckt sich bei ausgewarchsenen Tieren bis in die spitze der Eichel, bei jungen fehlt sie in der rorderen Eichel- 
hälfte bisweilen vollkommen. Die Abhängigkeit der Eichelhöhlengröße von der Fixierungsart wurde bereits erwähnt (Abschn. B), es hat also keinen Zweck, ihren Durchmesser in Zahlen wiederzugeben. Von der Spitze bis zum Beginn der Eichelorgane bleibt der Eichelhöhlenquerschnitt annähernd gleich groß, dann nimmt er ziemlich schnell an Größse zu. Ungefähr am caudalen Ende des Glomerulus erreicht die Eichelhöhle ihren größten Durchmesser (Abb. 9), dann verengt sie sich ziemlich rasch wieder (Abb. 10 und 11) und wird an ihrem caudalen Ende dorsal durch die Herzblase, ventral durch das Ventralseptum geteilt. Letzteres ist nur 10-15 $\|$ lang, die ventralen Eicheltaschen sind sehr reduziert. Die dorsalen Eicheltaschen sind auch nur kurz, erstrecken sich etwa $30 "$ " weiter nach hinten als die ventralen.

Von der linken dorsalen Eicheltasche schnürt sich die Eichelpforte $a b$ und mündet 100-150 $\leadsto$ hinter ihrem Beginn nach außen (Abb. $15 \mathrm{EP}$ ). In ihrem Verlauf wird sie sehr weit. Durch ihr etwa 10 " hohes, kubisches bis zylindrisches Epithel ist sie auf Quer- wie Längsschnitten stets sehr gut erkennbar. Bewimperung konnte ich auf ihrem Epithel nicht feststellen.

Glomerulus: Der laterale Glomerulus ist 150 bis 250 " lang, der ventrale etwas kürzer, ein dorsaler fehlt. Der Glomerulus reicht fast so weit nach vorn wie die Eichelorgane. Hinten verschwindet er erst kurz vor dem Auftritt des Ventralseptums.

Der Bau des Glomerulus (Abb.13) ist einfach; seine Zellen liegen offen in der Eichelhöhle. Distal sind die Glomerulusfalten teilweise untereinander verbunden. Die Glomeruluszellen sind klein und kubisch, ihre Kerne sind im Verhältnis zum Zelleib groß3. Die Durchblutung des Glomerulus ist stark (Abb. 13), in den peripheren Glomerulusteilen sind mit Sicherheit Gefäßanastomosen erkennbar.

Die Abb. 13 bringt noch folgendes zum Ausdruck: Das Blut im zentralen Blutraum färbt sich mit Orange-Erythrosin weniger intensiv als das der Glomerulusgefäße. Da nur in Ausnahmefïillen zentraler Blutraum und Glomerulusgefäße gleichzeitig gefüllt sind, konnte ich diesen Befund bei anderen Tieren nicht bestätigen. Er kann vielleicht die folgende Ansicht

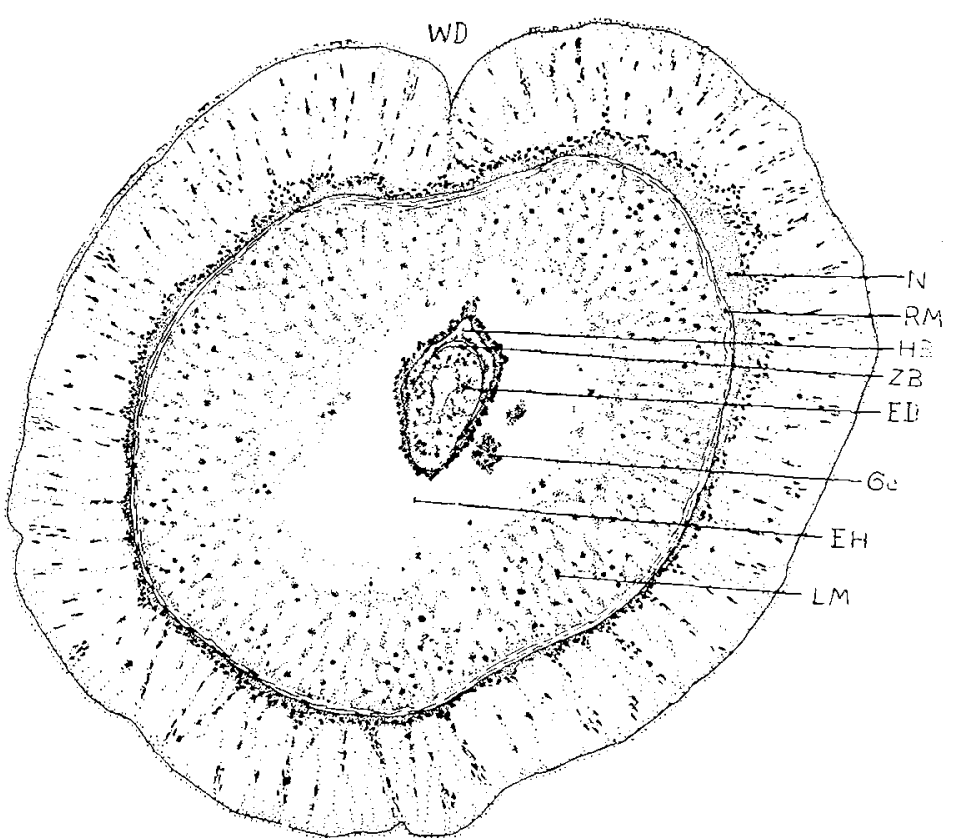

Abb. 9. Querschnitt durch den basalen Teil der Eichel, 250 " hinter Beginn der Eichelorgane. Behandelt, veryr. und beschriftet wie Abb. $8 . \quad \mathrm{ZI}=$ zentraler Blutraum.

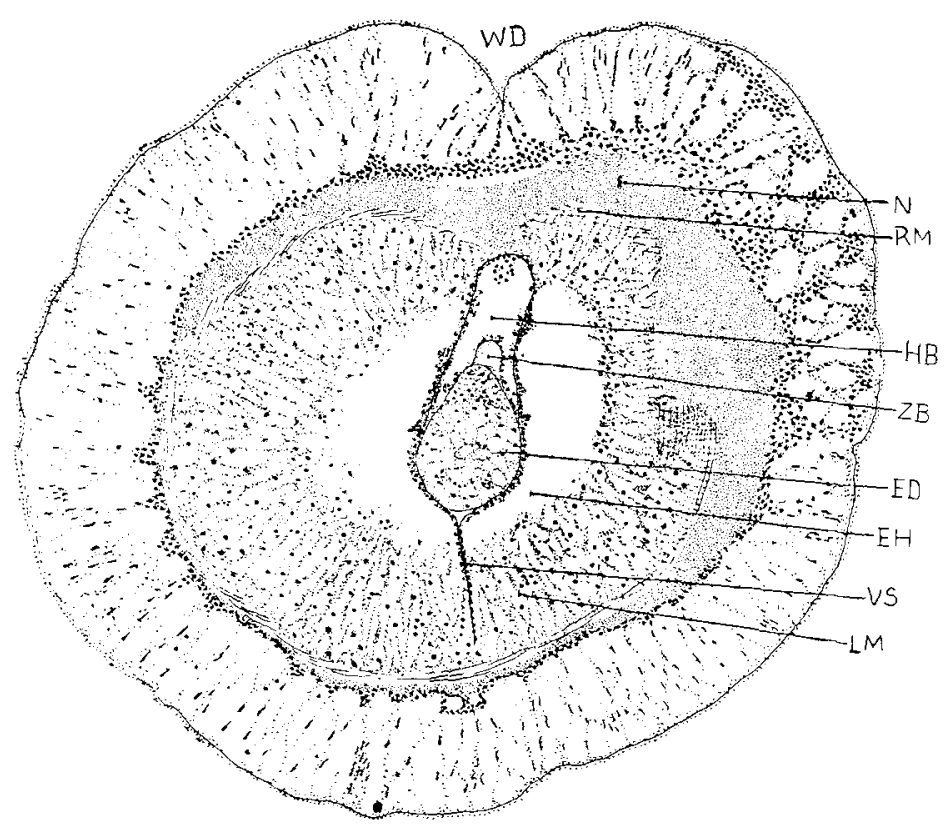

Abb. 10. Querschnitt durch den basalen Teil der Eichel, 300 " hinter Beginn der Eichelorgane. Behandelt, vergr. nud beschriftet wie Abb. 8 und 9. is $=$ Ventralseptum. 


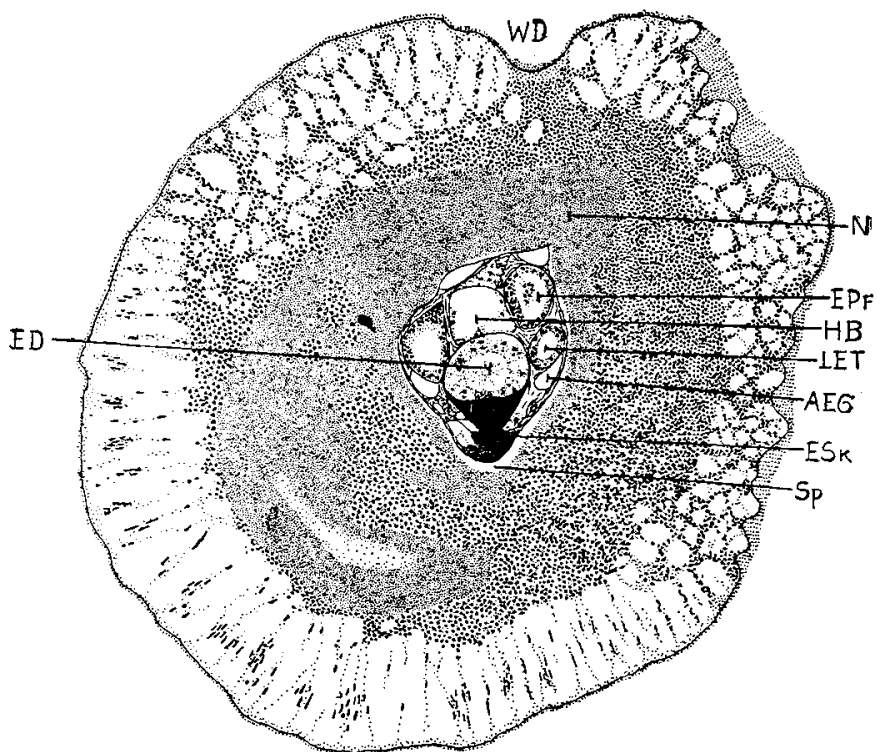

Abb. 11. Querschnitt durch den basalen Teil der Eichel, 350 " hinter Beginn der Eichelorgane. Behandelt, vergr. und beschriftet wie Abb. 8-10. AEG=abführendes Eichelgefäß, EPf = Eichelpforte, ESk = Eichelskelett, LET = linke dorsale Eicheltasche, $\mathrm{Sp}=$ Spaltraum, durch die Fixierung (?) entstanden.

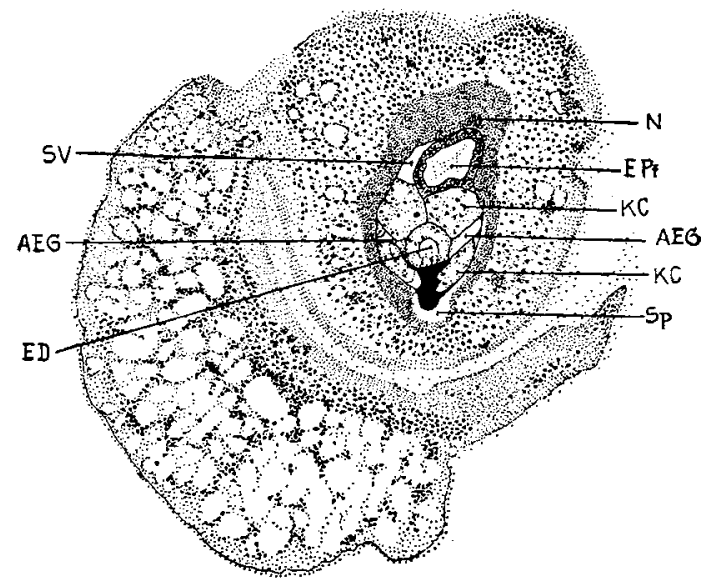

Abb. 12. Querschnitt durch den basalen Teil der Eichel, $400 \mu$ hinter Beginn der Eichelorgane.

Behandelt, vergr. und beschriftet wie Abb. $8-11 . \quad \mathrm{KC}=$ Kragencölom, SV $=$ Sinus venosus.

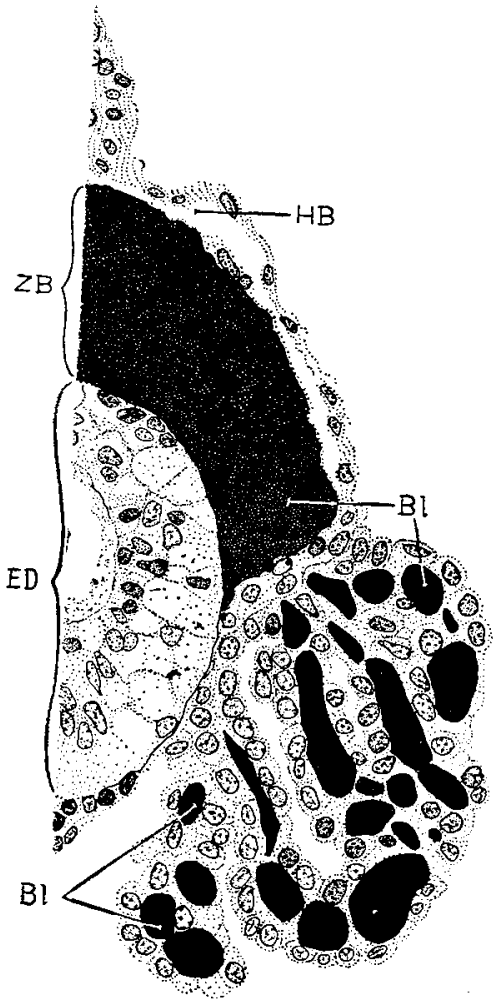

Abb. 13. Querschnitt durch die Eichelorgane eines kleinen Tieres, im hinteren Teil des Glomerulus (etwas hinter der Stelle, der Abb. 8 entnommen ist). Nark. Chloroform, fix. ZENKER, Fbg. TEO. Vergr. 603 fach. $\mathrm{Bl}=$ Blutgefäßre, $\mathrm{ED}=$ Eicheldarm, $\mathrm{HB}$ $=$ Herzblase, $\mathrm{ZB}=$ Zentraler Blutraum (gefüllt). Glomerulusdurchblutung gut erkennbar.
Batesons (nach Bronn S. 228) stützen: Das Blut wird im Glomerulus von Exkretstoffen befreit, die Exkretstoffe verlassen durch den Eichelporus die Eichelhöhle. Da nach BATESON in die Eichelhöhle von außen kein Wasser hineingelangt, die Exkretstoffe jedoch (mit Wasser) aus der Eichelhöhle hinausbefördert werden, muß angenommen werden, daß das Blut in den Glomerulusgefäßen konzentriert wird, wodurch die stärkere Färbbarkeit erklärt werden könnte

Herzblase: Die Herzblase ist je nach der Größe des Tieres und nach dem Kontraktionszustand der Eichel verschieden lang $(250-500 \mu)$. Auch die Größe und Form des Herzblasenquerschnitts ist nicht konstant. Sie kann auf Querschnitten als das größte Eichelorgan erscheinen.

Von allen Eichelorganen reicht die Herzblase am weitesten nach vorn. Dort wird sie von Epithelzellen überkleidet, zwischen denen sie auf Querschnittserien als rundlicher kleiner Zellhaufen erscheint. Dem direkt hinter ihr auftretenden Eicheldarm legt sie sich mit ihrer ventralen Seite an und bekommt dadurch, daß sich der Eicheldarm dorsalwärts in sie hineinschiebt, den typischen, stumpf halbmondförmigen Querschnitt (Abb. 8-10), den sie bis dicht vor ihrem hinteren Ende beibehält. Etwa in der Höhe der Eichelskelett-Endplatte wird die Herzblase durch die dorsalen Eicheltaschen von den lateralen Seiten des Eicheldarmes abgedrängt und bekommt eine ungefähr rechteckige Form (Abb. 11), nachdem sie kurz vorher an die dorsale Epidermis gestoßen war (Abb. 10). Die Herzblase reicht bis zum vorderen Ende der Kragencölome nach hinten, ihr caudales Ende wird von dem Sinus venosus (Verbindung des dorsalen Gefäßstammes von Rumpf und Kragen mit dem zentralen Blutraum) umspült. 
Die Herzblase ist ein blind geschlossener Sack. Sie ist von Epithelzellen ausgekleidet, die - vor allen Dingen an der ventralen Seite, sowie im vorderen Teil - sehr hoch werden können. Vorn enthält die Herzblase besonders viele Zellen, während ihr hinterer Teil auffälligerweise (vgl. BRonN S. 194) ziemlich leer ist (Abb. 11. In Abb. 14 ist die hintere Herzblasenwand flach angeschnitten).

Zwischen den die Herzblase vom Eicheldarm trennenden Grenzmembranen liegt der zentrale Blutraum (Abb. 9 und 13 ZB). In der ventralen Herzblasenwand konnte ich keinerlei Fasern nachweisen, sodaß die Annahme, sie fungiere mittels ihrer Muskulatur als Blutbewegungsorgan, schwer ist. Inwieweit Druck- und Salzgehaltschwankungen in der Herzblase und in der Eichelhöhle für die Blutbewegung eine Rolle spielen, ist eine offene Frage. Daß die Herzblase zu dem Blutgefäßsystem in Beziehung steht, ist bei vielen Enteropneusten sicher nachgewiesen und auch bei $S$. pygmaens wahrscheinlich.

Eicheldarm: Der Eicheldarm ist 500-750 " lang; durchschnittlich etwa in seiner Mitte tritt das Eichelskelett auf, sodaß der Eicheldarmkörper meist ebenso lang wie der Hals ist. Diese Maße variieren aber sehr, was u. a. auch mit der Art der Kontraktion des Tieres bei der Fixierung zusammenhängt. Das Tier, nach dem Abb. 6 gezeichnet ist, hatte einen auffällig langen Eicheldarmkörper.

Der Eicheldarm erstreckt sich nicht ganz so weit nach vorn wie die Herzblase, beide Organe werden an ihrer Spitze von Epithelzellen überkleidet. Auf Querschnitten durch die Eichel erscheint der Eicheldarmkörper rund bis entsprechend geringen Biegungen in seinem Verlauf oval. Er wird caudalwärts zunächst langsam und kontinuierlich, kurz vor Auftritt des Eichelskeletts ziemlich rasch dicker. Er erweitert sich dort taschenförmig nach der Ventralseite hin (Abb. 6), die Ventraltasche liegt der Endplatte des Skeletts auf. Letztere wölbt sich etwas in die Tasche vor. Dem Eicheldarmkörper liegt dorsal stets die Herzblase an, die großenteils auch seine Lateralseiten bedeckt. Seine Ventralseite und die ventralen Teile seiner Lateralseiten dienen als Ansatz für den Glomerulus. Die Ventraltasche des Eicheldarms stößt noch eben vor Auftreten des Eichelskeletts an die ventrale Epidermis.

DerEicheldarmhals liegt dorsal des Skeletts, hat dort zunächst noch ein sehr weites Lumen (Abb.14). Er wird weiter hinten im Eichelstiel sehr schmal und, wie die Abb. 15 und 20 er-

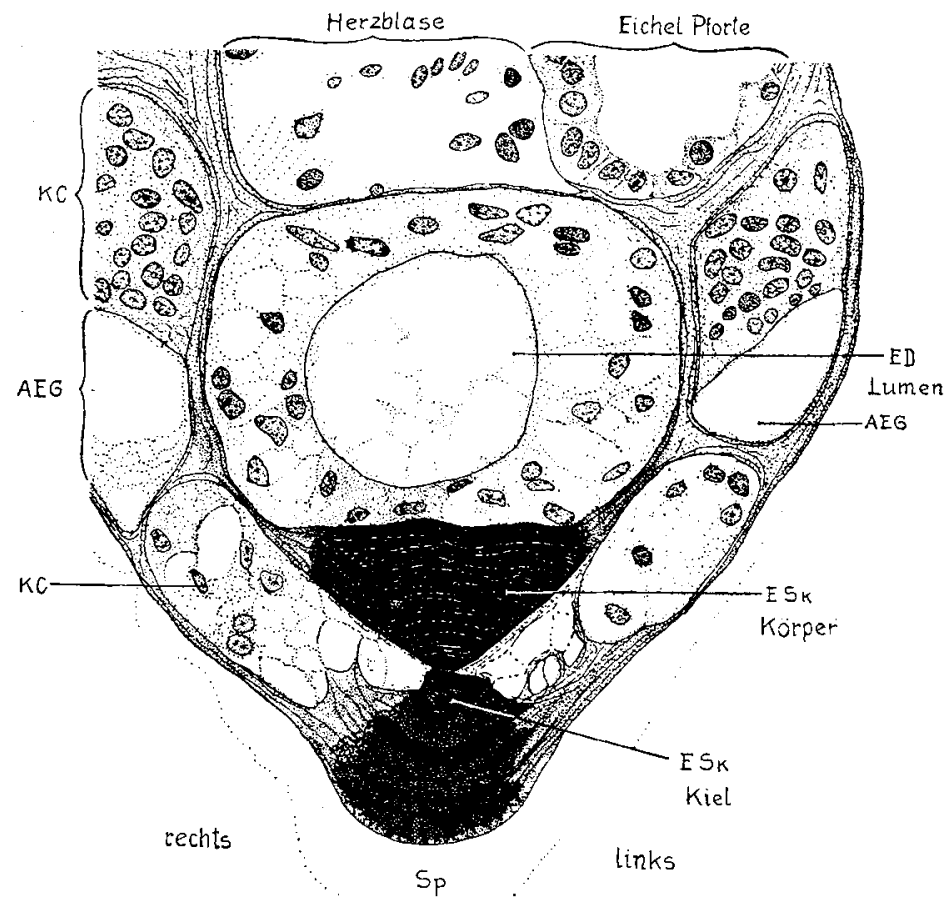

Abb. 14. Querschnitt durch die Eichelorgane bei Beginn des Eichelstiels. Behandelt wie Abb. 8 . Vergr. 603 fach. AEG $=a b-$ führende EichelgefäBe, EI)=Eicheldarm. Krageneölome (KC) hier anttretend, Eichel skelettschenkel bis fast hierher in den Kiel (ESk Kiel) erkennbar, $\mathrm{Sp}=$ Spaltraum zwischen Epidermis und Skelett (Fixierungsprodukt?). kennen lassen, bandförmig. $\mathrm{Er}$ umgreift teilweise auch die lateralen Seiten des Skeletts. Die Lateralseiten des Eicheldarmhalses werden zunächst von den Dorsaltaschen der Eichelhöhle, wenig weiter hinten von den Kragencölomen begrenzt (Abb.14). Letztere legen sich nach Verschwinden der Herzblase auch um die Dorsalseite des Eicheldarms (Abb. 15). Bis fast an den Eichelporus nach vorn reicht der Perihaemalraum, der von hier bis zur Mündung des Eicheldarms dessen dorsale Seite begrenzt (Abb.20).

Das Lumen des Eicheldarms erstreckt sich bis in seine Spitze. An der dicksten Stelle (Tasche) erreicht es einen Durchmesser von etwa $60 u$. Der Eicheldarmhals besitzt streckenweise kein Lùmen, kurz vor seiner Mündung tritt letzteres jedoch meist wieder auf.

Das Epithel des Eicheldarms ist stets wimperfrei. Im Körper und in der Ventraltasche ist es dick, hell vakuolisiert und ziemlich kernarm (Abb. 8-10, 13). Auf Längs- 
schnitten ist erkennbar, daß das der Skelettendplatte aufliegende Epithel des Eicheldarms dünn und drüsenarm ist. Im Eichelstiel wird das Epithel des Eicheldarms zunächst ventral, dann rundherum gleichmäßig dünn und drüsenarm (Abb. 15), was wohl damit zusammenhängt, daß die Eicheldarmzellen hier einen großen Teil des Eichelskeletts abscheiden. Die bei Enteropneusten allgemein beobachtete Erscheinung (vgl. BronN S. 51), daß skelettbildende Epithelien besonders niedrig sind, kommt also auch hier zum Ausdruck. Erst an der Mündungsstelle des Eicheldarms wird sein dorsales Epithel wieder hoch und hell vakuolisiert, so in das der Mundhöhle (Abb. 19 Ep III) übergehend.

Eichelskelett: Die Endplatte des Eichelskeletts ist sehr kurz. Thr vorderes Ende ist im Querschnitt etwa quadratisch, weiter hinten streckt sie sich jedoch sehr in dorsoventraler Richtung. Etwa $25 \mu$ hinter Beginn des Eichelskeletts werden seine Lateralseiten tief eingekerbt, sodaß der ventrale Kiel von dem dorsalen Körper getrennt wird (Abb. 11 und 14). Der Kiel ist meist dicker als der Körper, ist aber nur 20-40 $\mu$ lang. Er vereinigt sich also sehr bald wieder mit dem Körper, und das Skelett erhält nun einen parabelförmigen Querschnitt (Abb. 12). Letzterer wird caudalwärts bald flacher bis fast halbmondförmig (Abb. 15).

Weiter hinten wird der Querschnitt des Skeletts nun zunächst wieder rundlicher (Abb. 20), da die sein Inneres fast ganz ausfüllenden Schenkel in spitzem Winkel zueinander stehen. Caudalwärts rücken die Schenkel mehr und mehr auseinander, sodaß das

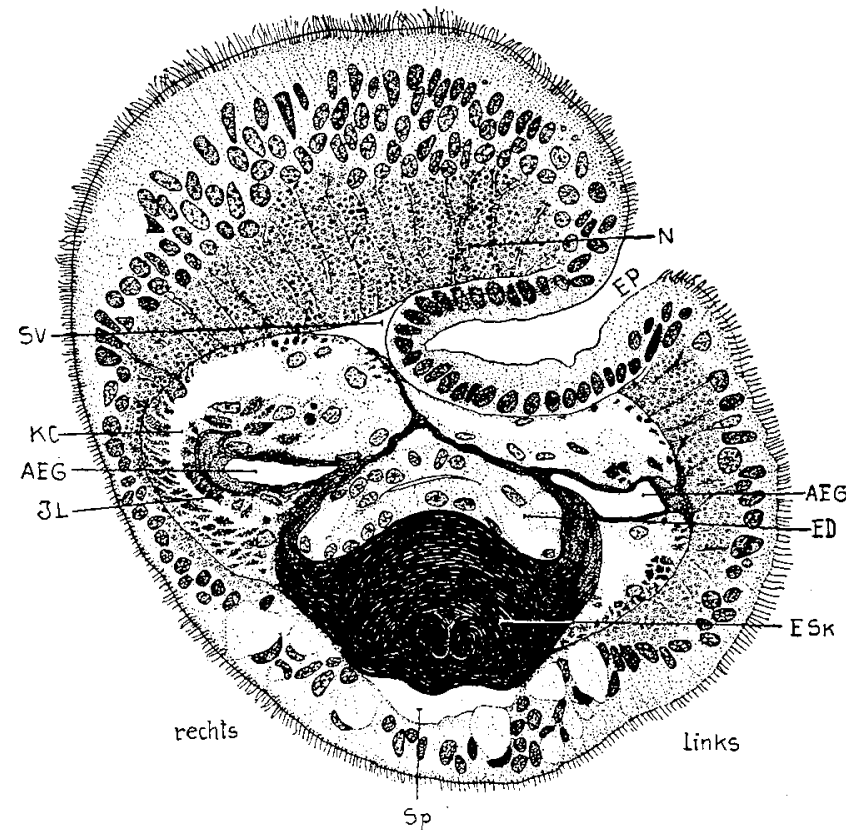

Abb. 15. Querschnitt durch den Eichelstiel in Höhe des Eichelporus (EP). Im Eichelskelett (ESk) die Skelettschenkel gut sichtbar. Behandelt und vergr. wie $\mathrm{Abb}$. 13. $\mathrm{AEG}=$ abführendes Eichelgefä $B, \mathrm{JL}=$ innere Längsmuskulatur des Kragens, $\mathrm{KC}=$ Kragencölom, $\mathrm{N}=$ Nervenfaserschicht, $\mathrm{SV}=$ Sinus venosus, $\mathrm{Sp}=$ Spaltraum (durch die Fixierung (?) entstanden). Skelett kurz vor der Mündung des Eicheldarms einen flachen, länglich ovalen Querschnitt bekommt. Die Skelettschenkel trennen sich in vorderen Drittel des Kragens voneinander. Sie reichen nicht ganz bis an das Hinterende des Kragens. Die Mundhöhle umfassen sie meist ungleichmäßig weit ventralwärts, was mit der meist unsymmetrischen Kontraktion des Kragens zusammenhängt.

Von ganz besonderem Interesse scheint es mir zu sein, daß die Eichelskelettschenkel, die sich im Inneren des unpaaren Teils des Eichelskeletts sehr weit cranialwärts verfolgen lassen (Abb.15), in den Kiel des Eichelskeletts reichen. Dem entspricht sehr gut eine Beobachtung an einem Jungtier: Der Körper des Eichelskeletts war hier nur ganz gering ausgebildet, während der Kiel bereits sehr mächtig war. Der Körper entsteht also sekundär. Chondroidgewebe, das bei allen Saccoglossusarten sehr schwach ausgebildet ist, konnte ich bei S.pygmaeus nicht sicher nachweisen. In der Skeletttaille zwischen Kiel und Körper, also dort, wo das Chondroidgewebe zu erwarten wäre, treten die Kragencölome als Zellhaufen zwischen Grenzmembranverdickungen auf (Abb. 14). Diese Grenzmembranverdickungen möchte ich für die Reste des chondroiden Gewebes halten. Auf allen meinen Schnittserien fiel mir ein Hohlraum zwischen Skelettkiel und Epidermis auf (Abb. 11, 12, 14 und 15, Sp), der wohl durch die Fixierung zustande kommt.

Im vorderen Teil des Eichelstiels wird das Skelett ventral von der Epidermis, lateral von den Kragencölomen und dorsal vom Eicheldarm begrenzt. Weiter hinten liegt es fast ganz zwischen Darmepithel (Abb. 20), die Skelettschenkel sind stets ganz von Darmepithel umgeben (Abb. 19).

\section{Kragen.}

Epidermis: Die Kragenepidermis erscheint auch auf Schnittpräparaten sehr einheitlich, weist keine besonderen Zonen auf (Abb. 6). Am Übergang in das Epithel der 
Mundhöhle wird sie niedriger, kernreicher und bekommt eine dickere Nervenschicht. Am Hinterende des Kragens wird bereits vor Übertritt des Kragenmarks in die Epidermis letztere zur Epidermis der Kiemenregion.

Die Kragenepidermis ist durchschnittlich $80 \mu$ hoch, davon entfallen auf die Nervenfaserschicht 3-10 $\mu$. Die Ausbildung eines Nervenringes am Hinterende des Kragens fehlt. Die Bewimperung ist, da Schleimzellen selten sind (s. u.), im Schnittpräparat deutlich. Die Dicke der Kragenepidermis variiert sehr, was vor allen Dingen davon abhängt, wie stark der Kragen aufgebläht ist. Besondere Charakteristika der Kragenepidermis (Abb. 16) sind folgende: Schleimzellen (S) sind selten, dafür kommen noch andere sezernierende Zellen ("Eiweißzellen“ E) in wechselnder Zahl und Ausbildung vor. Bei wenigen Tieren

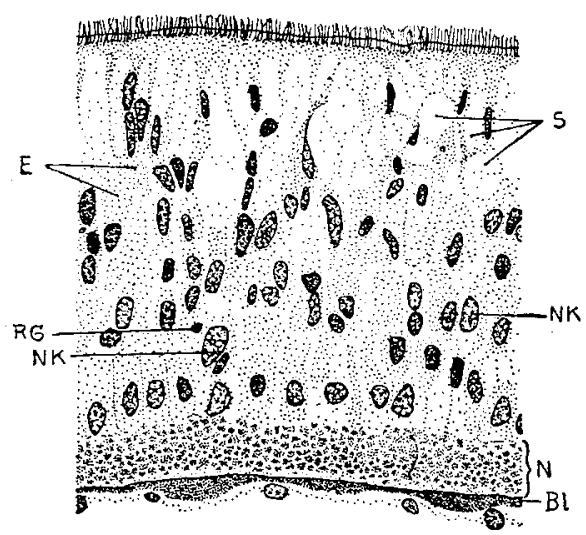

Abb, 16. Kragenepidermis. Aus einem Querschnitt durch den Mittelteil des Kragens. Behandelt und vergr. wie Abb. 7. Bl = Blutgefäße, $\mathrm{E}=$ Eiweißvakuolen, $\mathrm{N}=$ Nervenfaserschicht, NK $=$ Kerne des Nervensystems (?), $\mathrm{RG}=$ Granula, $\mathrm{S}=$ Schleimvakuolen.

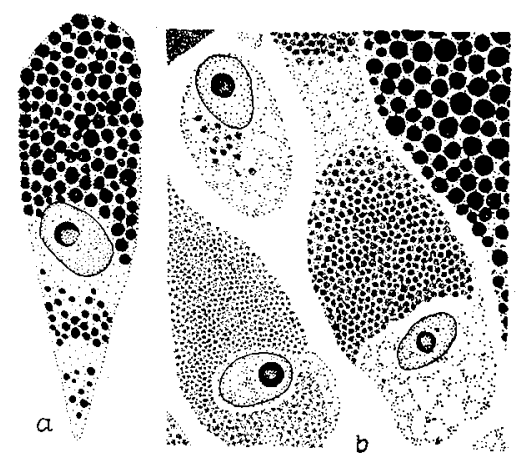

Abb. 17. Eiweißzellen der Epidermis des hinteren Teils des Kragens. a. Einzelne Zelle, b. Ausschnitł aus dem Epitbel. Nark. Chloroform, fix. CARNOY, Fbg. TEO, vergr. 2093 fach.

sah ich über die ganze Epidermis zerstreut lange schmale Vakuolen, die mit H. E. H. schwarz färbbare Granula enthielten. Bei den zwei mit CARNoY fixierten Tieren, die mir zur Verfügung standen, waren im hinteren Teil des Kragens noch andere, mit OrangeErythrosin lebhaft sich färbende, verschieden große Granula vorhanden (Abb. 17). Charakteristisch ist, daß im Gegensatz zu den Verhältnissen bei den Schleimzellen die Kerne sowohl in den ungranulierten als auch in den granulierten. Eiweißzellen weder in ihrer Lage noch in ihrer Form von dem Sekret beeinflußt werden. Dadurch ist es auch verständlich, daß im Gegensatz zur Eichelepidermis in der Kragenepidermis die Kerne über das ganze Epithel verstreut liegen. Einzelne große mit Eosin rot färbbare Granula (RG vgl. Eichelepidermis) sind auch in der Kragenepidermis vorhanden.

Kragenmark: Auf Querschnitten erscheint das Kragenmark oval bis bohnenförmig (Abb. 18 und 21), ventralseits bisweilen tief eingekerbt. Es ist etwa $100 \mu$ breit und $50 \mu$ hoch. Da es im kontrahierten Kragen natürlich nicht gerade von vorn nach hinten verläuft, ist es schwer, genaue Querschnitte zu bekommen. Es wird ventral von dem dorsalen Gefäßstamm (Abb. $18 \mathrm{DG}$ ), im hinteren Drittel des Kragens auch von den Perihämalräumen (Abb. 21) begrenzt. Übrigens liegt es frei in der Leibeshöhle.

Vordere und hintere Vorhöhlen, Centralkanal, dorsale Wurzeln und dorsaler Kiel fehlen. Ob Markhöhlen vorkommen, konnte ich nicht sicher feststellen, da sie von den ziemlich häufig vorhandenen Schleimvakuolen (Abb. $18 \mathrm{~S}$ ) nicht ohne weiteres unterscheidbar sind.

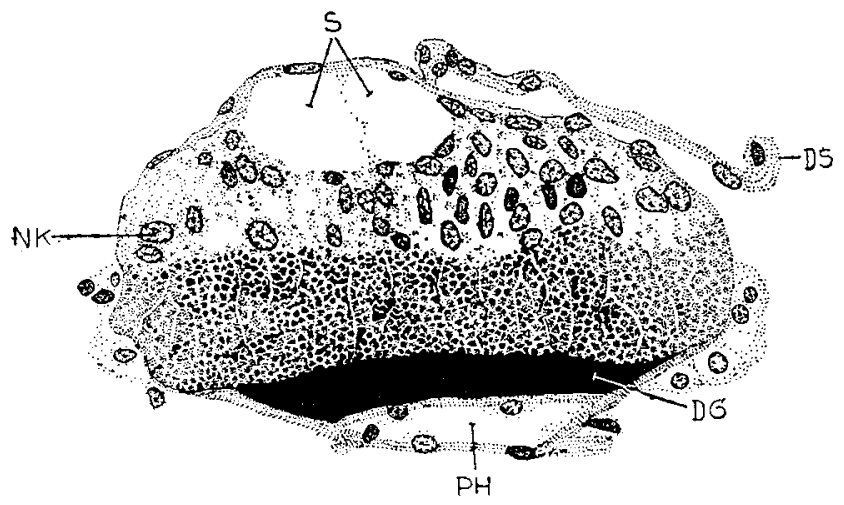

Abb. 18. Querschnitt durch Kragenmark, dorsalen Gefäßstamm (DG) und Perihämalraum (PH) etwa in der Mitte des Kragens. Behandelt und vergr. wie Abb. 7. $\mathrm{DS}=$ Dorsalseptum, NK= Kerne des Nervensystems (?), $\mathrm{S}=$ Schleimvakuolen . 
Der dorsale Teil des Kragenmarks enthält die Zellkerne, der ventrale die Nervensubstanz. Da ich auch hier - wie in der Körperepidermis - nichts von Zellgrenzen nachweisen konnte, konnte ich natürlich auch keine Riesenganglienzellen feststellen. Eine besondere, im Kragenmark häufige Kernart (groß, hell, mit rundem Nucleulus. Abb. 18 NK) scheint typisch für Nervengewebe zu sein. Sie kommt jedenfalls fast stets in Epithelien, die eine dicke Nervenschicht unter sich haben (Abb. 7 und 16), vor. Schleimvakuolen

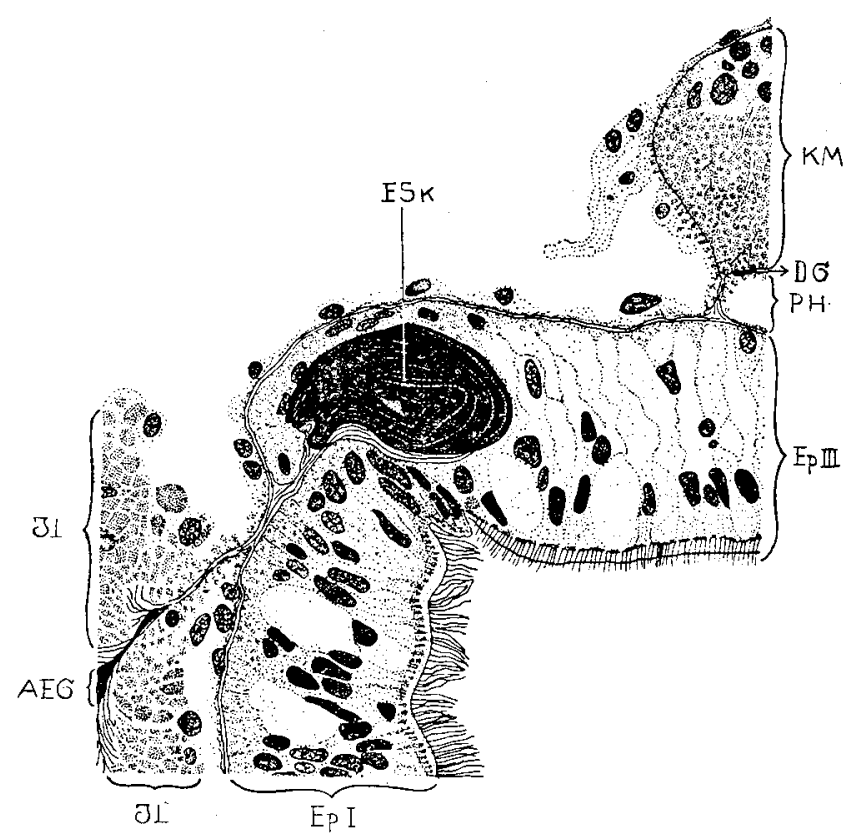

Abb. 19. Ausschnitt aus einem Kragenquerschnitt. Nark. Aethylurethan, fix. ZENKER, Fbg. WeIGERTs EH, vergr. 603 fach. $A E G=$ abtührendes Eichelgefäß, $\mathrm{DG}=$ dorsaler Gefäßstamm, JL :- innere Lüngsmuskulatur, $\mathrm{KM}=$ Kragenmark, $\mathrm{PH}--$ Perihämalraum. Die verschiedene Ausbildung des Nundhöhlenepithels (Ep I und Ep III) jederseits des Lichelskelettschenkels (ESk) erkennbar.

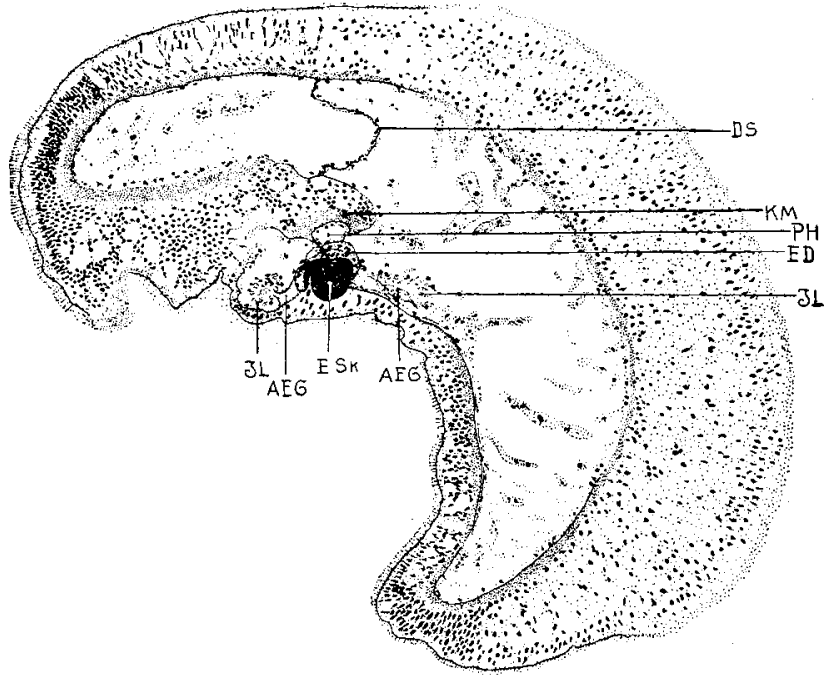

Abb. 20. Querschnitt durch den vordersten Teil des Kragens. Behandelt wie Abb. 19, vergr. 105 fach. AEG $=\mathbf{a b}-$ führendes Eichelgefäß, DS = Dorsalseptum, ED = Eicheldarm, ESk $=$ Eichelskelett, $\mathrm{JL}=$ innere Liingsmuskulatur, $\mathrm{K} M=$ Kragenmark, $\mathrm{PH}=$ Perihämalraum. sind im zelligen Teil des Kragenmarks öfters zu sehen (Abb. $18 \mathrm{~S}$ ). Faserstrukturen in dèrNervensubstanz konnte ich in "schlecht" fixierten Präparaten (Zellkerne deformiert, ohne deutliche Strukturen) besonders deutlich erkennen. Die Fasern verlaufen meist von der zelligen Schicht auf dem kürzesten Wege durch die Nervenschicht zur Grenzmembran.

Mundhöhle: Die Mundhöhle ist von folgenden gut unterscheidbaren Epithelarten ausgekleidet:

a) Der weitaus größte Teil der Mundhöhle (Abb. 19 Ep I, Abb. 21 und 22) besitzt ein durchschnittlich 35 " hohes Epithel, das viele kleine, mit Toluidin z. T. schwach, z. T. gar nicht färbbare, das ganze Epithel durchsetzende $\mathrm{Va}$ kuolen enthält. Basal zeigt dieses Epithel - wenigstens im vorderen Kragenteil (Abb. 20) - einen Cytoplasmasaum, der die Fortsetzung der Nervenschicht der Kragenepidermis darstellt. Inwieweit dieser Cytoplasmasaum nervöser Natur ist, ist natürlich nicht sicher zu sagen. Distal besitzt das Epithel einen feinen Cytoplasmasaum, in dem die Basalkörner der langen dünnen Wimpern liegen (Abb. 19). Die Kerne sind über das ganze Epithel zerstreut, am zahlreichsten sind sie im distalen Drittel.

b) Das die Skeletteile überkleidende Epithel gleicht dem der Ventralseite des Eichelstiels (s. d.), ist jedoch unbewimpert (Abb. 19).

c) Das Epithel zwischen den Eichelskelettschenkeln (Abb. 19 Ep III) ist $40-60$ " hoch, sehr stark vakuolisiert und ohne basalen Cytoplasmasaum. Es erstreckt sich cranialwärts in den Eicheldarm hinein, caudal geht es bereits vor dem Verschwinden der Eichelskelettschenkel in das normale Mundhöhlenepithel über.

Kragenpforten: Die Kragenpforten sind $70-100 \%$ lange ovale Kanäle (Abb. 6) mit - das ist sehr auffällig (vgl. BRONN S. 70, 71) - einfach ovalem Lumen (Abb.22). Sie verlaufen schräg von vorn dorsolateral nach hinten ventromedian. Ihr Epithel ist ca. $18 u$ hoch, proximal ganz mit Kernen gefüllt, distal kernfrei (Abb.22). Bewimperung konnte ich nicht nachweisen. 
Am Innenporus der Kragenpforten wird ihr Epithel plötzlich dünn, schlägt nach außen um und geht in das sie überziehende Cölomepithel über (Abb. 22). Der Übergang des hohen Epithels der Kragenpforte in das platte der ersten Kiementasche, in die die Kragenpforte mündet, ist unvermittelt.

Cölomverhältnisse: Die Kragencölome sind weite Räume, die sich bis fast zur Spitze des Eichelskeletts in den Eichelstiel erstrecken (Abb. 14 und 15 KC). Sie treten als Zellnester neben oder direkt anschließend an die hier verschwindenden Dorsaltaschen der Eichelhöhle auf und sind anfangs jederseits durch das abführende Eichelgefäß (AEG) in einen dorsalen und einen ventralen Teil getrennt. Der Ventralteil liegt lateral des Eichelskeletts (ESk), in der Bucht zwischen Kiel und Körper. Der linke Dorsalteil liegt neben Eicheldarm (ED) und Eichelpforte, der rechte neben Eicheldarm und Herzblase. Weiter hinten drängen sich die beiden Kragencölome zwischen Eicheldarm und Eichelpforte bzw. Sinus venosus (SV) und bleiben bis zum Auftreten des Perihämalraumes voneinander nur durch ein Septum getrennt (Abb. 15). Fast gleichzeitig verschmilzt lateral des abführenden Eichelgefäßes der dorsale und der ventrale Teil der Kragencölome miteinander, sodaß das Gefäß nur noch am Eicheldarm befestigt ist (Abb. 15 und 20).

Von ganz besonderem Interesse ist, daß die beiden Kragencölome durch ununterbrochene Septen völlig voneinander getrennt bleiben. Die Perihämalräume sind sehr schwach ausgebildet. Vorn, d. h. bereits im hinteren Drittel des Kragens, verschmelzen sie zu einem einheitlichen Raum. Dieser läßt sich dann allerdings bis in den Eichelstiel (Abb.20PH), und zwar fast bis zum Eichelporus verfolgen. Durch die sehr schräge Stellung des Kragen-Rumpf - Septums (Abb. 21 und 22 KRS) sind Peripharyngealräume angedeutet (PPh), erstreckt sich andererseitsdas Kragencölom dorsolateral in die Kiemenregion hinein (Abb. 22).

Der Kragen ist der weitaus muskelärmste Körperteil. Deutliche Stränge bildet eigentlich nur die innere Längsmuskulatur (Abb.

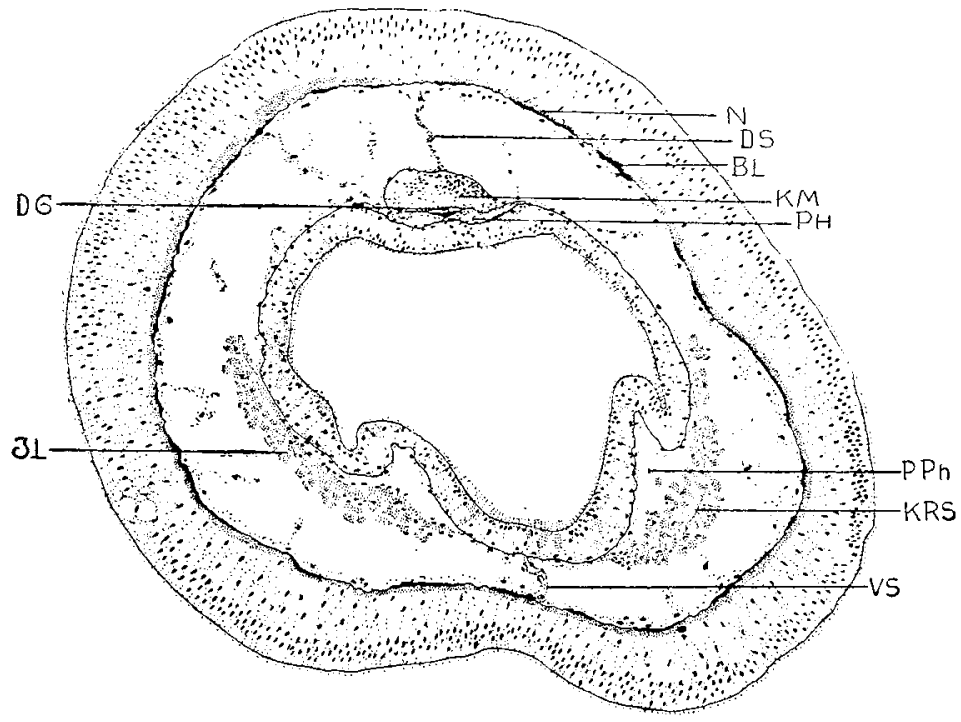

Abb. 21. Querschnitt durch den hinteren Teil des Kragens. Behandelt wie Abb. 7, vergr. 105 fach. $B L=$ Blutgefäße, $D S=$ Dorsalseptum, I)G = dorsaler Gefäßstamm, $\mathrm{JL}=$ innere Längsmus kulatur, KRS $=$ Kragenrumpfseptum, $\mathrm{N}=$ Nervenfaserschicht. Perihämalraum (PH) geteilt, mit Längsmuskelfasern. Peripharyngealräume (PPh) vorhanden. Ventralseptum (VS) schon sehr kurz.

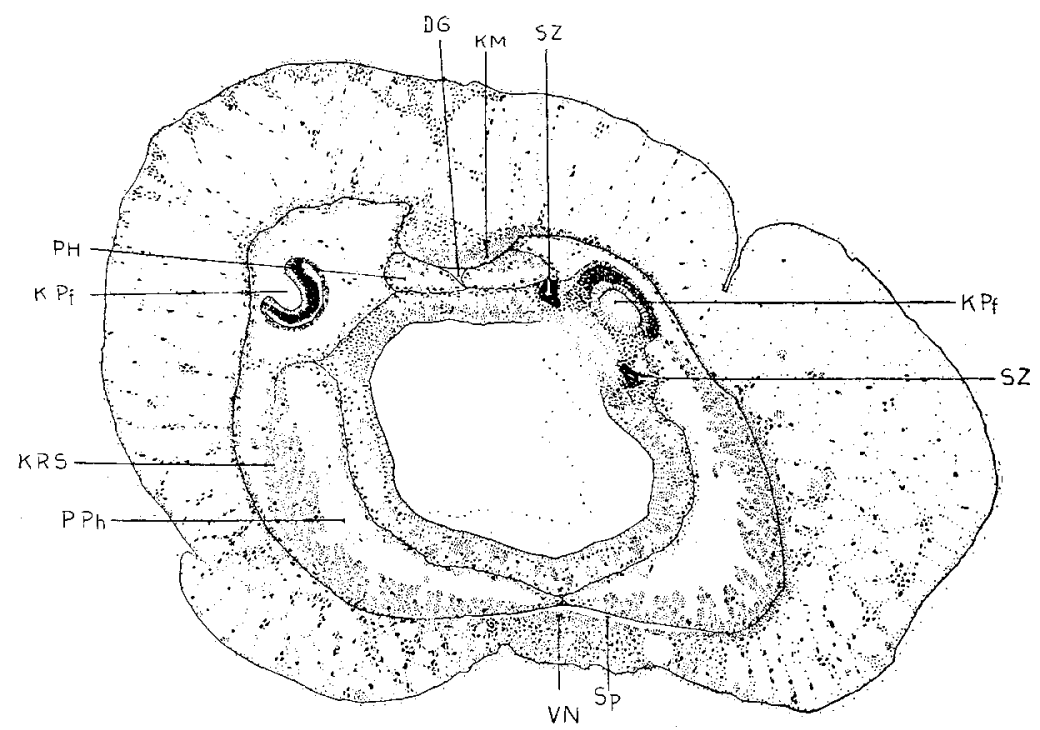

Abb. 22. Querschnitt đurch die Übergangszone vom Kragen zur Kiemenregion. Behandelt und vergr. wie $\mathrm{Abb}, 21$. DG $=$ dorsaler Gefäßstamm, KPf $=$ Kragenpforte, $\mathrm{KRS}=$ Kragenrump ${ }^{s}$ septum, $\mathrm{PH}=$ Perihämalraum, $\mathrm{PPh}=$ Peripharyngealraum, $\mathrm{Sr}_{\mathrm{r}}=$ Spaltraum (Fixierungsprodukt), $\mathrm{SZ}=$ Septalzinke der erste Tiemenspalte. Epidermis bereits die der Kiemenregion, $K_{\ldots} . .$, enmark (KM) epithelial, ventraler Nervenstamm (VN) deutlich, Ventralseptum verschwunden. 
19, 20, $21 \mathrm{JL}$ ), welche vom Kragenrumpfseptum (Abb. $22 \mathrm{KRS}$ ) längs der abführenden Eichelgefäße (AEG) bis in die vorderste Spitze der Kragencölome (Abb. 15) hineinreicht. Im hinteren Teil des Kragens weisen auch die Perihämal- und Peripharyngealräume Längsmuskelfasern auf (Abb. 21 und 22). Von Ring- und Radialfasern ist im Kragen fast nichts nachweisbar, unter dem Epithel des Körpers und der Mundhöhle befinden sich nur ganz feine Faserlagen.

Vereinzelte freie Zellen sind stets im Kragencölom zu finden, bei dem Tier, dem die Abb. 20 entnommen ist, waren sie außergewöhnlich zahlreich.

\section{Rumpf.}

Epidermis: Die Rumpfepidermis weist Schleim- und Eiweißdrüsen auf. Entsprechend den Beobachtungen am lebenden Tier finden sich die Schleimvakuolen vorwiegend dorsalseits, dort namentlich in der Kiemenregion enorm mächtig (Abb. 23). Sie sind dort nach Fixierung oft derart gequollen, daß der dorsale Nervenstamm als tiefe Rinne erscheint, und daß von den warzenfreien Streifen, die die Poren umgeben (Abb. 4), nichts mehr vorhanden ist. Letzteres ist nur so zu erklären, daß die Schleimzellen dieser Epidermisteile beim lebenden Tier nicht als Warzen erscheinen, sich auch nicht mit Neutralrot färben. Es ist klar, daß die Körperkontraktion und die Quellung des Schleims bei der Fixierung auch kleine Schleimvakuolen deutlich hervortreten lassen muß.

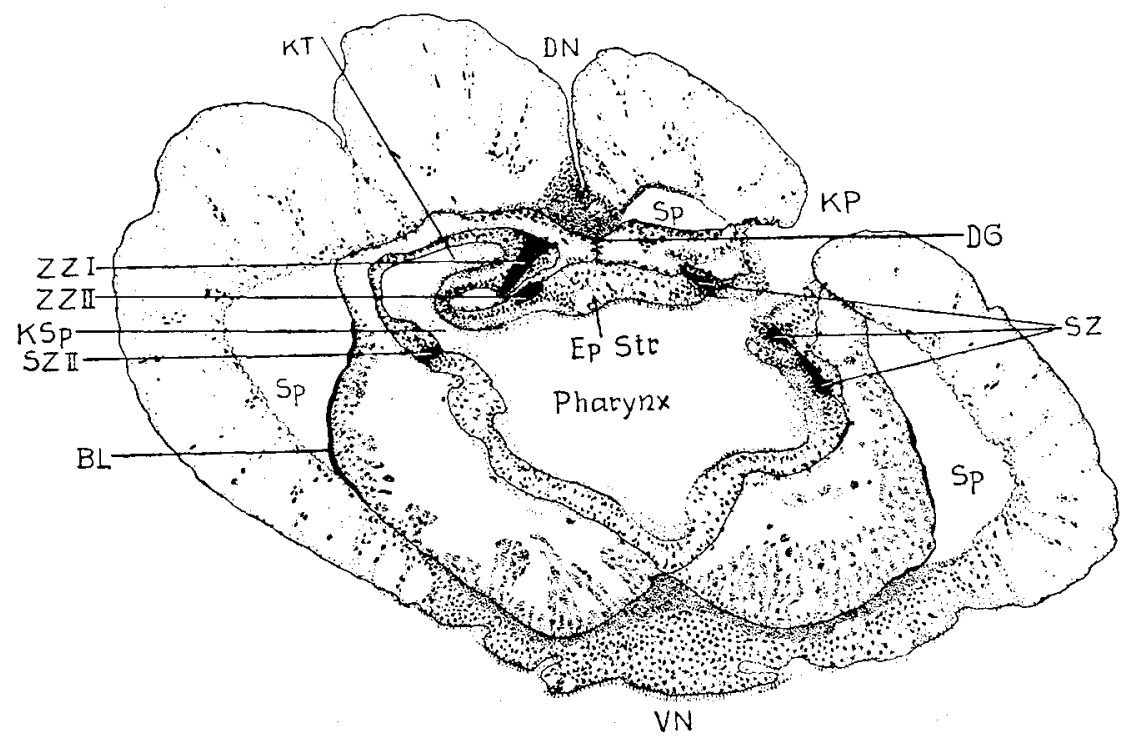

Abb. 23. Querschnitt durch die Kiemenregion. Behandelt und vergr. wie Abb. 21.

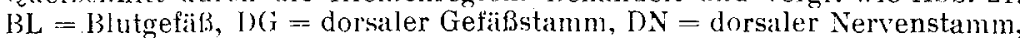
EpStr $=-=$ Epibranchialstreifen, $\mathrm{KP}^{\prime}=$ Kiemenporus, $\mathrm{KSp}=$ Kiemenspalte $\mathrm{K}^{\mathrm{T}}=\mathrm{K}$ Kiementasehe, $\mathrm{SP}=$ durch Quellung der Schleimzellen hervorgerufene Spalten, $\mathrm{SZ}=$ Septalzinke, $\mathrm{VN}=$ ventraler Nervenstamm, ZZ = Zungenzinke. SZII und ZZII hängen zusammen.

Auch auf den postbranchialen Rumpfregionen sind die schleimzellhaltigen Epidermispartieen bedeutend höher als die ziemlich niedrige Epidermis. Auffällig ist die starke Ausbildung der Schleimvakuolen über den Gonaden, deren Ausführungsgänge dadurch sehr undeutlich sind. In der Endregion (Abb. 28) ist dies anscheinend nicht mehr so sehr der Fall, ich habe aber von diesem Körperteil nur sehr wenige Schnittserien, und nur bei einer ist die Epidermis intakt. Da der Füllungszustand der Schleimvakuolen sehr wechselnd ist, muß man, um ein Bild von der durchschnittlichen Mächtigkeit der Schleimdrüsen zu bekommen, natürlich mehrere Serien untersuchen.

Die Eiweißvakuolen sind kleiner als die Schleimvakuolen, rundlich oval, und mit homogenem, mit Eosin oder Orange-Erythrosin färbbarem Inhalt. In der Endregion sind sie anscheinend selten.

Im dorsalen - nur beim Übergang vom Kragen zur Kiemenregion ist das anders (Abb. 22) - und ventralen Nervenstamm sind sowohl Schleim- als auch Eiweißvakuolen spärlich bis fehlend, die Schleimvakuolen stets klein (Abb. 23 und 26). Die nervöse Sub- 
stanz reicht etwa bis in die Mitte der Zellen. Zwischen der Nervenmasse und dem distalen Cytoplasmasaum liegen sehr viele Kerne dicht nebeneinander. Der dorsale Nervenstamm wird in den hinteren Rumpfregionen schwächer, bleibt aber erhalten (Abb. 28).

Die ventrale Seite des Rumpfes ist stets deutlich bewimpert, über dem dorsalen Nervenstamm konnte ich die Bewimperung nicht einwandfrei nachweisen. Über den Schleimvakuolen habe ich niemals Wimpern gesehen.

Pharynx: Kiemen- und Nahrungsteil des Pharynx sind nicht voneinander getrennt. Da die Kiemenspalten ziemlich horizontal liegen, ist der Querschnitt des Pharynx, wie auf Abb. 23 zu sehen ist, etwa dreieckig, die Spitze des Dreiecks zeigt ventralwärts.

Das Epithel des breiten Epibranchialstreifens (Abb. $23 \mathrm{Ep}$ Str) ist nicht besonders modifiziert. Es ist, ebenso wie das des ventralen Pharynx, sehr ähnlich dem der Mundhöhle, nur meist etwas niedriger und einfacher. Im hinteren Teil des Pharynx ähnelt sein Epithel sehr dem des Oesophagus.

Die Kiemenspalten haben den für Enteropneusten (wenigstens Harrimaniiden) typischen Bau. Die Abb. 4 zeigt sie in der Aufsicht, Abb. 23 die Kiemenregion im Querschnitt (Kiemensepten und -zungen längsgeschnitten), Abb. 24 die Kiemenregion im diagonalen Längsschnitt (Septen und Zungen quergeschnitten). Die Abb. 25 gibt ein schematisches Bild von der Lage der Kiemenskelette $(\mathrm{SZ}+\mathrm{ZZ}) \mathrm{zu}$ den Kiemenspalten (K Sp), wie man es sich beim lebenden Tier vorzustellen hat. ${ }^{1}$ )

Die eigentlichen Kiemenspalten sind die Uförmigen Öffnungen des Darms (K Sp), die durch die Kiemensepten (KS) voneinander getrennt sind. Die U-Form der Kiemenspalten wird durch die Kiemenzungen (KZ) gebildet, die von dorsomedian in die Kiemenspalten hineinragen. Die Kiemenspalten sind durch einen zur Kiementasche sich erweiternden Kanal mit dem epidermalen Kiemenporus verbunden. Kiemensepten und -zungen sind durch Skelette (SZ, ZZ) gestützt.

Die Kiemenskelette haben die Form dreizinkiger Gabeln. In der Aufsicht (Abb. 25) erscheinen sie breit und füllen die Septen und Zungen abgesehen von der Zungenhöhle (ZH) fast ganz aus. Die Abb. 24 läßt erkennen, daß sowohl die Septal- (SZ) als auch die Zungenzinken (ZZ) ziemlich schwach, fast blattförmig sind, ferner, daß die Septalzinken deutlich zweiteilig sind. An ihrem freien Ende weichen die Septalzinken nur wenig auseinander (Abb. 25). Die Zungenzinken (ZZ) reichen, im Gegensatz zu den Verhältnissen bei Protoglossus Koehleri, bis in die Zungenspitzen.

Die außerordentliche Deformierbarkeit gerade der Kiemenregion besagt $u$. a., daß die Skelettsubstanz sehr elastisch ist. Die ausgeprägte Längsteilung der
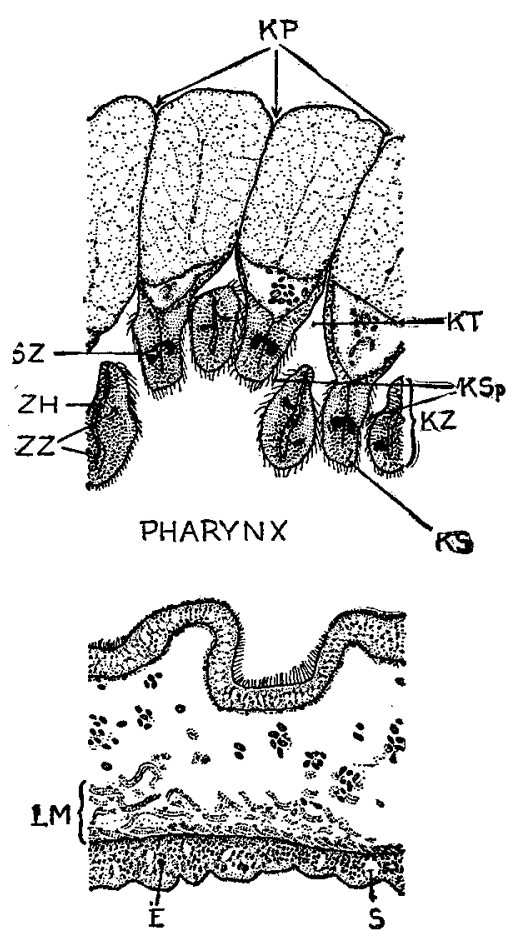

Abb. 24. Diagonaler Längsschnitt durch die Kiemenregion. Behandelt und vergr. wie $\mathrm{Abb}$. 20. $\mathrm{E}=$ Eiweißvakuole, KS = Kiemenseptum, KSp $=$ Kiemenspalte, $\mathrm{KT}=$ Kiementasche, $\mathrm{KZ}=$ Kiemenzunge, LM = Längsmuskulatur, $\mathrm{S}=$ Schleimvakuole, $\mathrm{SZ}=$ Septalzinke, $\mathrm{ZH}=$ Zungenhöhle, $\mathrm{ZZ}=$ Zungenzinke.

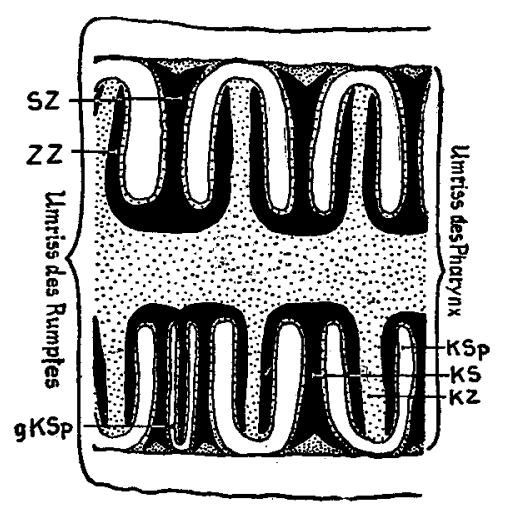

Abb. 25. Darstellung der Form der Kiemenskelette und ihrer Lage zu den Kiemenspalten (KSp), Kiemensepten (KS) und Kiemenzungen $(\mathrm{KZ})$, von dorsal gesehen. Vergr. 67 fach. gKSp $\rightleftharpoons$ geschlossene Kiemenspalte, $\mathrm{SZ}=$ Septalzinke, $\mathrm{ZZ}=$ Zungenzinke.

1) Da die Kiemenspalten bei der Fixierung stets besonders deformiert werden, sind sie in dieser Form aus Schnittserien nicht rekonstruierbar. Die Länge und Breite der Zungen- und Septalzinken, sowie die Form des freien Endes der Septalzinken und die Art der Verbindung der Septalzinken mit den Zungenzinken sind jedoch in Schnittserien erkennbar, die richtige Form der Kiemenzungen und -spalten ist nur beim lebenden Tier zu sehen. Die Abb. 25 ist aus verschiedenen Beobachtungen rekonstruiert. 
Septalginken (Abb. 24) mag auch dazu beitragen, daß die Kiemenspalten beweglich sind. Bei Kontraktion der Kiemenspalten (Abb. 4 und $25 \mathrm{~g} \mathrm{KSp,} \mathrm{sowie} \mathrm{Abb.} \mathrm{24)} \mathrm{rücken} \mathrm{die}$ Zungenzinken hintereinander.

Die letzten Kiemenspalten, bei jungen Tieren bisweilen alle, sind sehr einfach gebaut (Abb. 1 und 2), namentlich ihre Zungen sind weitgehend rückgebildet. Die Skelette sind aber auch hier deutlich vorhanden.

Das Epithel der Kiemensepten (KS) und -Zungen (KZ) ist etwa $20 \mu$ hoch und trägt außerdem noch sehr lange und zarte Wimpern. Die Kerne liegen basal in ein bis mehreren sehr dichten Lagen, distal findet sich feines, gestreiftes Cytoplasma. Schleimvakuolen sind sehr selten und klein. Die Kiementaschen (KT) besitzten ein einschichtiges plattes bis

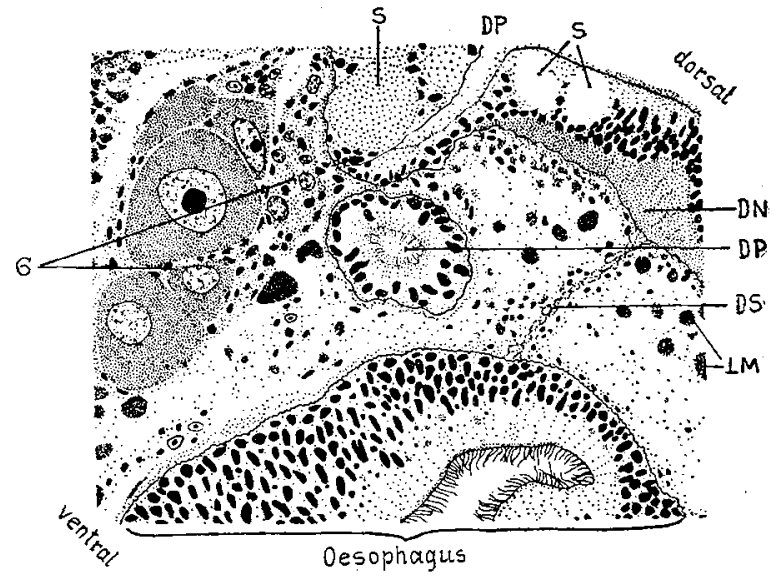

Abb. 26. Cuerschnitt (schräg) durch den Ösophagus, an der Ausmündungsstelle einer Darmpforte (DP). Nark. Chlorotorm, fix. HELLY, Fbg. TEO, vergr. 267 fach. D N = dorsaler Nervenstamm, DS Dorsalseptum, $\mathrm{G}=$ zwei Gonaden ( $($, nur die eine mit großen Eiern), LM = Längsmuskelfasern, $\mathrm{S}=$ Sehleimvakuolen.

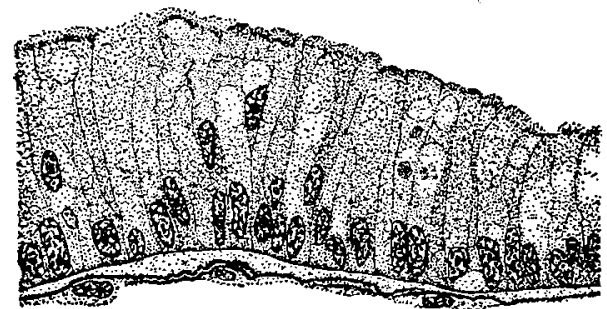

Abb. 27. Längsschnitt durch das Epithel des Leberdarms (erster Teil). Behandelt und vergrößert wie $A b b .19$. Unter dem Epithel deutlich Muskel (?) -fasern erkennbar. kubisches, wimperfreies Epithel, das nur vereinzelt Schleimvakuolen besitzt. Es ist etwa $6 \mu$ hoch. Von der Epidermis ist es scharf abgesetzt, in das der zugehörigen Septen geht es kontinuierlich über (23 und 24$)$.

Ö so phagus: Der Ösophagus ist nicht sehr dick und schwach geschlängelt. Sein Epithel (Abb. 26) erscheint dadurch, daß es in der Diek̀e sehr variiert $(20-60 \mu)$, gewellt. Es ist stets kontinuierlich bewimpert, die Kerne liegen basal in mehreren sehr dichten Lagen. Die Distalseite des Epithels ist kernfrei und hell vakuolisiert, die Vakuolen erscheinen bisweilen dureh Granulationen auch dunkler.

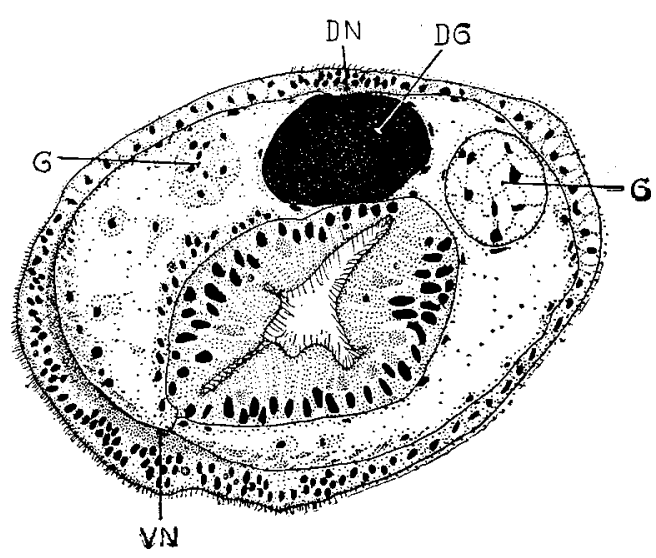

Abb. 28. Querschnitt durch die Endregion eines kleinen Tieres. Behandelt wie Abb. 13, vergr. 267 fach. $D G=$ dorsaler Gefäßstamm (gefüllt), $\mathrm{DN}=$ dorsaler Nervenstamm, $\mathrm{G}$ $\stackrel{=}{=}$ Gonade, $\mathrm{VN}=$ ventraler Nervenstamm. Muskulatur bei diesem Tier aborm schwach.

Im caudalen Teil des Ösophagus konnte ich bei den meisten Tieren (nicht bei allen) ein Darmpfortenpaar nachweisen. Diese Darmpforten (Abb. 26 DP) sind klein und führen vom Darm dorsolateral nach außen. Sie münden fast direkt neben dem dorsalen Nervenstamm. Ihr Epithel ist kubisch, einschichtig und bewimpert. Die sie umgebende Grenzmembran ist häufig etwas verdickt („Darmpfortenskelett“).

Leberdarm: Im ersten Teil der Leberregion ist der Darmumfang gering, der Darm intensiv geschlängelt, die Darmwand etwa so dick wie die des Ösophagus. Im zweiten Teil ist dagegen der Darmumfang meist sehr groß, sodaß der Darm die Leibeshöhle fast ganz ausfiullt, die Darmwanddicke gering (Abb. 1-3).

Das Epithel des Leberdarms (Abb. 27) ist wimperfrei und besitzt - dadurch ist es auf den ersten Blick vom Ösophagusepithel unterscheidbar - auch dort, wo es sehr 
dick ist, nur eine Lage großer Kerne. Diese Kerne liegen meist basal, reichen jedoch bisweilen weit distalwärts. Im zweiten Teil liegen sie infolge der geringeren Epitheldicke weiter auseinander und sind rundlicher als im ersten. Ventralseits findet sich im Leberdarmepithel eine längsverlaufende Furche.

Enddarm: Der Darm der Caudalregion (Abb. 28) besitzt ein kubisches bis zylindrisches, bewimpertes Epithel.

Leibeshöhlenorgane: In der Kiemenregion berührt der Darm ventral direkt die Epidermis (Abb. 23), erst im hinteren Teil der Kiemenregion tritt ein kurzes Ventralseptum auf. In den postbranchialen Rumpfregionen ist stets ein Ventralseptum vorhanden.

Die Längsmuskulatur ist im Rumpf ziemlich stark, ihre Stärke variiert jedoch sehr. Bei dem kleinen Tier, dem die Abb. 28 entnommen ist, ist sie extrem schwach. Verschieden verlaufende Fasern unter dem Körper- und Darmepithel sind bisweilen sichtbar (Abb. 27).

Die Gonaden sind bei größeren Tieren zu jeder Jahreszeit sehr auffällig. Im November und Dezember fixierte $0^{x} o^{x}$ hatten die Hoden prall mit Spermatozoen gefüllt. Die Eier in den weiblichen Gonaden sind zu jeder Jahreszeit ziemlich groß. (Das Tier, dem die Abb. 26 entnommen ist, ist am 5. IV. fixiert). Gonadenausführgänge habe ich auf Schnittpräparaten niemals einwandfrei gesehen.

\section{F. Diagnose.}

A eußere Gestalt: Gesamtlänge $30 \mathrm{~mm}$; Länge der Eichel beim lebenden Tier bis $7 \mathrm{~mm}$, größte Breite $1 \mathrm{~mm}$. Kragenlänge $1 \mathrm{~mm}$, größte Breite $1,3 \mathrm{~mm}$. Eichel im Querschnitt rund, mit feiner Kontur längs der dorsalen Mittellinie. Nach Fixierung erscheint diese Kontur als Furche in der Epidermis. Kragen mit einer Ringfurche. Kiemenporen und -spalten gut sichtbar, 9-23 Paar. Gonaden treten erst am Ende der Kiemenregion auf und kommen von da an in allen Körperregionen vor. Genitalwülste sehr gering ausgebildet. $\sigma^{x}$ Gonaden als leuchtend rote Flecke sichtbar. Eier zu jeder Jahreszeit vor allem aber während der Ablagezeit (November und Dezember) - sehr groß und sehr dotterreich. Dorsalnervenstamm des Rumpfes in der Kiemen- und Leberregion deutlich, caudalwärts schwächer werdend. Ventraler Nervenstamm in der Kiemenregion noch nicht sichtbar, in den hinteren Rumpfregionen deutlich. Keine ventralen Muskelwülste vorhanden. In der Epidermis aller Körperteile - nur auf dem Kragen fast fehlend - warzenförmige. Hautstrukturen in z. T. charakteristischer Anordnung. Farbe: Eichel milchig-hellgelb, Kragen braungelb, Kiemenregion hellgelb, caudalwärts farblos werdend. Postbranchiale Rumpfregionen farblos mit schwarz, bräunlich oder grünlich durchschimmerndem Darm.

Eichel: Nervenfaserschicht in der dorsomedianen Längskontur verdickt. Ringmuskelschicht dünn, etwas dicker als die Nervenfaserschicht, nahe der Eichelbasis keinen Sphinkter bildend. Längsmuskelfasern nicht in konzentrischen Schichten angeordnet. Eichelhöhle bis in die vordere Spitze der Eichel reichend, dort nicht erweitert. Ventrales Eichelseptum äußerst kurz, sich von dem caudalen Ende der ventralen Cölomsäcke nur wenig nach vorn erstreckend. Letztere bereits bei Beginn des Eichelskeletts verschwindend. Nur die linke dorsale Cölomtasche mit einer weiten Eichelpforte in Verbindung; Porus links. Der Eicheldarmkörper hat ein ununterbrochenes Lumen, das sich vor Beginn des Skeletts ventralwärts erweitert. Lumen des Eicheldarmhalses mehrfach unterbrochen. Herzblase groß. Glomerulus nur lateral und ventral der Eichelorgane vorhanden. Eichelskelett mit gering ausgebildeter Endscheibe, Skelettschenkel nicht ganz bis zum Hinterende des Kragens reichend. Chondroides Gewebe fast fehlend.

Kragen: Keine verschiedenen Epidermiszonen. Dorsales und ventrales Mesenterium ununterbrochen vorhanden. Kragenpforten im Querschnitt oval, mit dicker, kernreicher Wand. Perihämalräume bereits im hinteren Drittel des Kragens miteinander verschmelzend, nicht ganz bis zum Eichelporus nach vorn reichend. Peripharyngealräume im hinteren Drittel des Kragens vorhanden. Kragenmark ohne Vorhöhlen, dorsale Wurzeln und dorsalen Kiel. Markhöhlen nicht sicher vorhanden, Schleimvakuolen im Kragenmark häufig. Im caudalen Teil des Kragens kein Nervenring. Die Mundhöhle ohne besondere dorsale Blindtasche, Eicheldarmmündung im vorderen Teil des Kragens.

Rumpf: Pharynxlumen einheitlich. Kiemenspalten fast horizontal liegend. Epithel auf der breiten Epibranchialrinne nicht modifiziert. Kiemenzungen und Kiemensepten mit hohem, kernreichem Wimperepithel, Kiementaschen mit niedrigem, wimperfreiem Epithel. Pharynx im vorderen Teil der Kiemenregion direkt an die ventrale Epidermis stoßend. Ösophagus sehr kurz und einheitlich, mit hohem Wimperepithel und basal in 
mehreren Schichten liegenden Kernen. Im vorderen Teil des Ösophagus noch die letzten Kiemenspalten, im hinteren Teil ein Paar Darmpforten. Leberdarm sehr lang, mit wimperfreiem Epithel, dessen Kerne nur eine Lage bilden. Meist zwei Leberdarmteile unterscheidbar: Ein vorderer, dickwandiger und englumiger und ein hinterer dünnwandiger und weitlumiger. Endregion lang, mit mitteldickem Darm. Letzterer mit hohem Wimperepithel.

Vorkommen: „Amphioxusgrund“, ein grobsandiges, detritusarmes Sediment. $4-5$ Meilen NO von Helgoland.

\section{Schriftenverzeichnis.}

Bravibell, F. II. Rogers und H. A. Cole, Occurence of an Enteropneust. Nature 133 (1934).

TAN DER Horst, C. J., Die Enteropneusten aus den Meeren der U.S.S.R. Zool. Jb. Anat. 58 (1934).

- Hemichordata. Bronns Klassen und Ordnungen des Tierreichs, Bd. 4, Abt. t, 2. Buch (1927-1937).

FREDA FROOTKO KAPELUS, The Anatomie of the Enteropneust Saccoglossus inhacensis sp. n. Ann. of the Natal Museum 8, Part I (1936).

Hixichs und JicoBr, Saccoglossus pygmaeus, eine neue Enteropneustenart aus der südlichen Nordsee. Zool. Anz. Bd. 121. 1938.

Steler, A., Sil unculoidea, Phoronidea, Brachiopoda Enteropneusta und Acrania vol Alexandrien in Aegypten. Notizen des I)eutsch-Italienischen Institutes für Meeresbiologie in Rovigno d'Istria 1936.

Alle weitere Literatur siehe in Bronns Klassen und Ordnungen des Tierreichs. 